\title{
Color Subspaces as Photometric Invariants
}

\author{
Todd Zickler, Harvard University \\ Satya P. Mallick, University of California, San Diego \\ David J. Kriegman, University of California, San Diego \\ Peter N. Belhumeur, Columbia University
}

\begin{abstract}
Complex reflectance phenomena such as specular reflections confound many vision problems since they produce image 'features' that do not correspond directly to intrinsic surface properties such as shape and spectral reflectance. A common approach to mitigate these effects is to explore functions of an image that are invariant to these photometric events. In this paper we describe a class of such invariants that result from exploiting color information in images of dichromatic surfaces. These invariants are derived from illuminant-dependent 'subspaces' of RGB color space, and they enable the application of Lambertian-based vision techniques to a broad class of specular, non-Lambertian scenes. Using implementations of recent algorithms taken from the literature, we demonstrate the practical utility of these invariants for a wide variety of applications, including stereo, shape from shading, photometric stereo, material-based segmentation, and motion estimation.
\end{abstract}

\section{Introduction}

An image is the product of the shape, reflectance and illumination in a scene. For many visual tasks, we require only a subset of this information, and we wish to extract it in a manner that is insensitive to variations in the remaining 'confounding' scene properties. For 3D reconstruction, for example, we seek accurate estimates of shape, and we design systems that are insensitive to variations in reflectance and illumination.

One practical approach to these problems is to compute a function of the input images that is invariant to confounding scene properties but is discriminative with respect to desired scene information. A number of these invariants are described in the literature, with the simplest example being a normalized-RGB image. For a Lambertian scene, the normalized RGB color vector at each pixel depends on the 
spectral reflectance of the corresponding surface patch but not its orientation with respect to a light source. It is useful invariant for material-based segmentation.

Like normalized-RGB, most existing invariants seek to isolate information about the material properties in a scene and are therefore designed to be invariant to local illumination and viewing geometry. In contrast, this paper considers a class of invariants that deliberately preserve geometry information in a way that is invariant to specular reflections. The proposed invariants provide direct access to surface shape information through diffuse shading effects, and since diffuse shading is often well approximated by the Lambertian model, they satisfy the 'constant-brightness assumption' underlying most approaches to stereo reconstruction and structure-from-motion. In addition, these invariants provide access to surface normal information, which can be recovered using Lambertian-based photometric reconstruction methods.

The idea underlying the proposed invariants can be interpreted geometrically. When the illuminant color is known, and the reflectance of surfaces can be represented by a dichromatic model (Shafer, 1985), we can linearly transform the space of RGB tristimulus vectors in a way that isolates specular reflection effects. Following the transformation, two sensor channels are free of these effects, and this two-dimensional "color subspace" constitutes a specular invariant. Since this operation is linear, the diffuse shading information is preserved by the transformation, and the invariant can be exploited photometrically. Also, the method places no restrictions on scene texture because the computation operates independently at each image point. Finally, it only requires knowledge about the spectral content of scene illumination and therefore makes no assumptions about the spatial distribution of light sources.

This paper begins with the case of RGB images and singly-colored illumination environments (Sect. 3), in which case the linear transformation can be interpreted as a transformation to an alternative, illuminant-dependent color space. We refer to this space as $S U V$ color space. In addition to providing a specular invariant, we show that this color space leads naturally to a notion of generalized hue (Sect. 3.1). We are not limited to this case, however, and a similar procedure can be shown to handle mixed-illumination environments and hyper-spectral images (Sect. 4). To assess the utility of the proposed invariants they are applied in number of visual tasks, including binocular stereo, shape-from-shading, photometric stereo, optical flow estimation, and segmentation (Sect. 5). In each of these cases, when the source colors are known, significant improvements result from computing the invariants as a pre-process. 


\section{Background and Related Work}

As mentioned in the introduction, many existing invariants seek to isolate information about material properties in a scene. One such property is surface reflectance, which is often described by the bi-directional reflectance distribution function, or BRDF. Here, we consider the BRDF to be a five-dimensional function of wavelength and imaging geometry, and we write it $f(\boldsymbol{\theta}, \lambda)$, where $\boldsymbol{\theta}=\left(\theta_{i}, \phi_{i}, \theta_{r}, \phi_{r}\right)$ encodes the directions of the incident and reflected radiance in the local coordinate system. The simplest model of reflectance is the Lambertian model, according to which the BRDF is a constant function of the imaging geometry, so that $f(\theta, \lambda)=f(\lambda)$.

A number of photometric invariants have been proposed for Lambertian scenes. Normalized-RGB, $r-g$ chromaticity, and hue/saturation images are all examples of representations that are independent of diffuse shading (the geometric relation between a surface normal and the illumination direction) and depend only on the spectral reflectance of the surface and the spectral power distribution (SPD) of the illuminant. Additional invariants to either local geometry or spectral reflectance can be computed from "reflectance ratios" when multiple images of a scene are available (e.g., Wolff and Angelopoulou, 1994), or when the reflectance of the surface is spatially coherent (e.g., Nayar and Bolle, 1996); an invariant to both local geometry and illuminant spectral power distribution (SPD) can be computed from a single image under appropriate imaging conditions (Hordley et al., 2002).

Invariants for more general scenes, including some scenes with specularities, can be derived from the Shafer's dichromatic model of reflectance (Shafer, 1985). According to this model, the BRDF of the surface can be decomposed into two additive components: the interface (specular) reflectance and the body (diffuse) reflectance. In theory, by separating an image according to these components, one can obtain invariants to either diffuse or specular reflection effects.

According to the dichromatic model (with the neutral interface assumption (Lee et al., 1990)), the observation of a surface point can be written

$$
e_{k}=\sigma_{d} d_{k}+\sigma_{s} s_{k}
$$

where $\sigma_{d}$ and $\sigma_{s}$ are geometric scale factors that depend on the material properties and the local view and illumination geometry $(\boldsymbol{\theta})$, and

$$
\begin{aligned}
d_{k} & =\int E(\lambda) R(\lambda) C_{k}(\lambda) d \lambda \\
s_{k} & =\int E(\lambda) C_{k}(\lambda) d \lambda .
\end{aligned}
$$


Here, $E(\lambda)$ is the SPD of the incident illumination, $R(\lambda)$ is the spectral reflectance of the surface, and $C_{k}(\lambda)$ is the spectral sensitivity of a linear sensor. A typical RGB camera yields three such observations, and in this case we write $\mathbf{e}_{R G B}=$ $\left\{e_{k}\right\}_{k=R, G, B}$ and define $\mathbf{d}=\left\{d_{k}\right\}_{k=R, G, B}$ and $\mathbf{s}=\left\{s_{k}\right\}_{k=R, G, B}$ to be the diffuse color and specular color, respectively. These are conventionally assumed to be vectors of unit length.

There is practical utility in separating the diffuse and specular components in an image. Since diffuse reflections are typically well-represented by the Lambertian model, computing this separation as a pre-process allows the application of powerful Lambertian-based vision algorithms to a variety of non-Lambertian scenes. Materials that can be treated in this way include plant leaves, cloth, wood, and the skin of fruits (Lee et al., 1990; Tominga and Wandell, 1989) in addition to a large number of dielectrics (Healey, 1989). The dichromatic BRDF model has also proven useful for a number of applications involving human skin (e.g., face recognition (Blanz and Vetter, 2003), pigment-based analysis and synthesis (Tsumura et al., 2003)), even though the reflectance of human skin is more accurately described by the higher dimensional BSSRDF (Wann Jensen et al., 2001).

Despite its apparent utility, image analysis relying on explicit decomposition of the diffuse and specular components is rare because the separation problem is ill-posed. Classically, this separation problem is addressed using color histogram analysis. As made clear by Eq. 1, in the RGB cube, a collection of color vectors from a dichromatic material under multiple view and illumination configurations (i.e,., different values of $\boldsymbol{\theta}$ ) lie in the dichromatic plane - the plane spanned by the specular and diffuse colors, $\mathbf{s}$ and $\mathbf{d}$ (Shafer, 1985). These color vectors often cluster in the shape of a 'skewed-T' in this plane, where the two limbs of the skewed-T correspond to diffuse and specular reflections (Gershon, 1987; Klinker et al., 1988). When these limbs are sufficiently distinct, the diffuse and source vectors can be recovered, the two components can be separated, and the highlights can be removed (Klinker et al., 1988).

While this method works well for homogeneous, dichromatic surfaces in the noiseless case, there are two significant limitations that make it difficult to use in practice. First, many surfaces are textured and violate the homogeneous assumption. Even when an image does contain homogeneous surfaces, a non-trivial segmentation process is required to identify them. Second, in order for the specular and diffuse limbs of the skewed-T to be distinct, the specular lobe must be sufficiently narrow (i.e., its angular support must be small relative to the curvature of the surface.) Overcoming these restrictions generally requires additional assumptions regarding spatial coherence on the surface (Nayar et al., 1997; Mallick et al., 2006; Tan and Ikeuchi, 2003; Tan et al., 2006), specific parametric models for specular reflectance (Ragheb and Hancock, 2001), or the use of multiple images that exploit 
additional cues such as polarization (Wolff and Boult, 1991; Nayar et al., 1997).

When the source color is known and constant over a scene, one can compute specular invariants that are based on transformations of RGB color space and do not require explicit specular/diffuse separation. This is the approach taken in this paper, and it is related to the work of Tan and Ikeuchi (2003), who obtain such a specular invariant using a source-dependent non-linear transformation of the RGB values at a pixel. The transformation is computed independently at each point, and it yields a positive grayscale image that depends only on diffuse reflections ( $\sigma_{d}$ and d) and is independent of specular effects $\left(\sigma_{s}\right)$. Another non-linear transformation that provides a similar invariant under white illumination is proposed by Yoon and Kweon (2006a). As an alternative, Park (2003) defines a linear transformation that provides two channels that, while not pure invariants, are highly insensitive to specular reflections. Following this transformation, the measurements in one channel correspond predominantly to specular reflectance information, while the other two are predominantly diffuse. Unlike these existing methods, we present true invariants that are computed linearly, and hence have the unique property of preserving diffuse shading (and geometry) information.

The invariants presented in this paper assume knowledge of the scene illuminant s. In controlled environments, or when the illuminants do not change significantly over time, the required source color vectors can be measured by imaging a calibration target. This is the approach taken in this paper. While not explored here, it may be possible to apply these invariants in more uncontrolled environments by combining them with existing image-based methods for illuminant estimation. For scenes with sufficient color diversity, for example, one can estimated the illuminant color using statistical knowledge of common sources and surfaces (Brainard and Freeman, 1997; Finlayson, 1996; Finlayson et al., 2001; Lehmann and Palm, 2001; Rosenberg et al., 2001; Sapiro, 1999; Tominga and Wandell, 2002), and for glossy scenes with only a small population of diffuse colors, it can be estimated using methods based on the dichromatic model (Finlayson and Schaefer, 2001; Lee, 1986; Tan et al., 2004; Tominga and Wandell, 1989). The accuracy of these methods depends on the materials and illuminants that are present in a particular scene, so in a generic setting, one would probably want to use some combination of them. For discussions, and for detailed evaluations of some of these algorithms, the reader is referred to (Barnard et al., 2002a,b; Hordley and Finlayson, 2006).

Invariants for scenes with more general reflectance functions are developed by Narasimhan et al. (2003). They describe a general model of reflectance consisting of a product of a "material" term (Lambertian albedo, Fresnel coefficient, etc.) and a "geometry" term that encodes the relationship between the surface normal, light-source, and viewing direction. Invariants to both of these terms can be computed from either multiple observations of a single point under variable view or 
illumination, or from one observation of a spatially-coherent scene. The geometry invariant is of particular interest, since it can be used directly for material-based segmentation.

\section{A Source-dependent Color Space}

Suppose we treat RGB tristimulus values as points in $\mathbb{R}^{3}$ and linearly transform the RGB coordinate system by rotating the axes. Also, as shown in the left of Fig. 1, suppose this rotation is such that one of the axes (red, say) becomes aligned with the direction of the effective RGB source vector $\mathbf{s}$. This transformation defines a new color space (see below), which we refer to as the $S U V$ color space. It can be defined according to $\mathbf{e}_{S U V}=\mathbf{R e}_{R G B}$ using any $\mathbf{R} \in S O(3)$ that satisfies $\mathbf{R s}=(0,0,1)$. From Eq. 1 it follows that tristimulus vectors in the transformed space satisfy

$$
\mathbf{e}_{S U V}=\left(\overline{\mathbf{d}} \sigma_{d}+\overline{\mathbf{s}} \sigma_{s}\right)
$$

with

$$
\overline{\mathbf{d}}=\mathbf{R d}, \text { and } \overline{\mathbf{s}}=\mathbf{R} \mathbf{s}=(0,0,1) .
$$

Notice that according to our definition, the $S$ channel is uniquely defined for a given $\mathbf{s}$ (and thus a given illuminant SPD and sensor), while the $\mathrm{U}$ and $\mathrm{V}$ channels can be arbitrarily chosen from the family of orthonormal bases for the plane orthogonal to s.

The SUV color space is a source-dependent color space because it depends on the effective source color vector in the image. It has two important properties. First, it separates the diffuse and specular reflection effects. The $\mathrm{S}$ channel encodes the entire specular component and an unknown fraction of the diffuse component, while the remaining two channels ( $\mathrm{U}$ and $\mathrm{V}$ ) are independent of $\sigma_{s}$ and are therefore specular invariants.

The second important property is that shading information is preserved by the linear transformation. This is clear from Eq. 4. If $\mathbf{r}_{i}^{\top}$ denotes the $i^{\text {th }}$ row of $\mathbf{R}$, the values of the two diffuse channels satisfy

$$
e_{U}=\mathbf{r}_{1}^{\top} \mathbf{d} \sigma_{d} \text { and } e_{V}=\mathbf{r}_{2}^{\top} \mathbf{d} \sigma_{d} .
$$

Assuming Lambertian diffuse reflectance, $\sigma_{d}$ is a constant function of the local view and illumination directions. In this case, the two-channel color vector

$$
\mathbf{j}=\left(e_{U}, e_{V}\right)
$$

and its monochromatic relative

$$
j=\sqrt{e_{U}^{2}+e_{V}^{2}}
$$



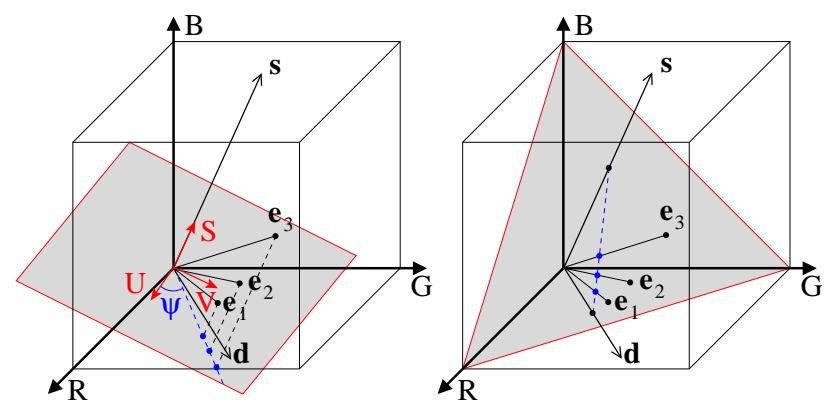

Figure 1: Linear and non-linear transformations of the RGB cube. Three observations of the same material yield color vectors $\mathbf{e}_{1}, \mathbf{e}_{2}, \mathbf{e}_{3}$ in the dichromatic plane spanned by the source and diffuse colors $\mathbf{s}$ and $\mathbf{d}$. Left: The SUV color space is defined by a rotation of the RGB coordinate vectors. One axis is aligned with the source color, and two of three resulting channels (UV) are invariant to specular reflections. Diffuse shading information is preserved in these channels and can be used to recover shape. Additionally, the ratio between the $\mathrm{U}$ and $\mathrm{V}$ channels represents generalized hue $(\psi)$, which provides a second invariant depending only on spectral reflectance. Right: Unlike SUV space, central projection used to compute $r$ - $g$ chromaticity values and HSV-type color spaces does not preserve diffuse shading information.

provide direct information about the normal vector on the surface, with the terms $\mathbf{r}_{2}^{\top} \mathbf{d}$ and $\mathbf{r}_{3}^{\top} \mathbf{d}$ in Eq. 5 contributing to the effective Lambertian albedo values.

An example of the monochromatic specular invariant computed from SUV space is shown in Fig, 2. In this example, the invariant was computed using the source color determined by intersecting lines in chromaticity space (Lee, 1986), and then transforming the image from RGB to SUV space on a pixel-by-pixel basis. (Here, we choose $\mathbf{R}=\mathbf{R}_{G}\left(-\theta_{\mathbf{s}}\right) \mathbf{R}_{B}\left(\phi_{\mathbf{s}}\right)$ where $\mathbf{R}_{k}(\theta)$ is a right-handed rotation about the $k$-axis by angle $\theta$, and $\left(\theta_{\mathbf{s}}, \phi_{\mathbf{s}}\right)$ are the elevation and azimuthal angles of the source vector $\mathrm{s}$ in the RGB coordinate system.) Comparing the result to the original image, we see that specular effects are largely removed. Note that the dichromatic model is violated when saturation occurs in the input images, and this causes errors at points of extreme brightness.

To see that SUV space is in fact a color space, recall that any linear color space can be defined by a linear transformation of the color matching functions of another. Such a transformation provides a mapping, say, between the ISO RGB color space (with an identified white point) and the CIE XYZ color space, and it induces a corresponding invertible linear mapping between the tristimulus vectors in the two spaces. The rotation matrix described above is a coordinate transformation, and it therefore defines a spectral space that is related to the original sensor space 

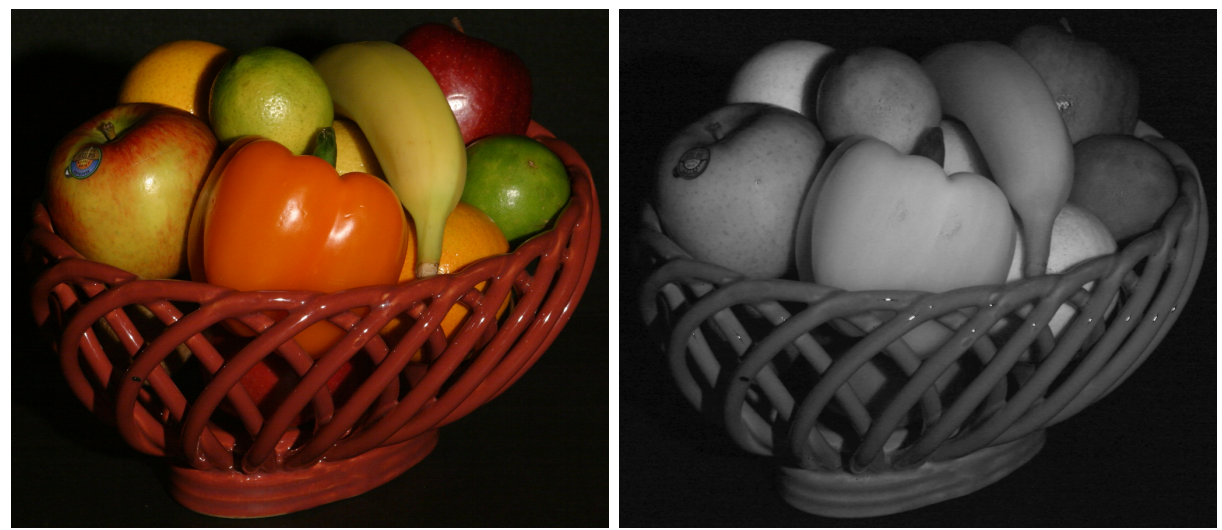

Figure 2: Input RGB image (left) and its corresponding specular invariant (right) computed pixel-wise according to Eq. 7 using the known illuminant color.

through a corresponding linear transformation of the sensor sensitivity functions. The important point is that following the transformation, the illuminant SPD $E(\lambda)$ integrates to black against two of the three transformed sensitivity functions. Thus, by converting to SUV space, we are implicitly choosing a transformation of the sensor such that the transformed sensitivities $\bar{C}_{k}$ satisfy

$$
\int E(\lambda) \bar{C}_{k}(\lambda) d \lambda=0, \quad k=1,2 .
$$

It is clear that the same invariant properties could be obtained using any transformation $\mathbf{T} \in G L(3)$ satisfying $\mathbf{T s} \propto[0,0,1]^{\top}$. The rotation matrix used in the definition above is simply one practical choice.

Figure 1 compares the linear, source-dependent SUV color space with conventional non-linear representations of color that also have invariant properties. Nonlinear representations such as $r-g$ chromaticity and hue-saturation-value (HSV) are computed by central projection. Each RGB vector in the RGB cube is intersected with the plane $R+G+B=c$ for some constant $c$. For example, hue and saturation correspond to the distance and polar angle of these intersection points relative to the cube diagonal, and chromaticity coordinates are derived from the intersection of these color vectors with the plane $R+G+B=1$. Non-linear representations such as these are useful for recognition, for example, because they remove Lambertian shading and shadow information. All positive scalar multiples of $\mathbf{e}_{R G B}$ map to the same chromaticity coordinates and the same hue.

In contrast, the diffuse channels of SUV color space preserve diffuse reflection effects encoded in the geometric scale factor $\sigma_{d}$. Since diffuse reflectance is 

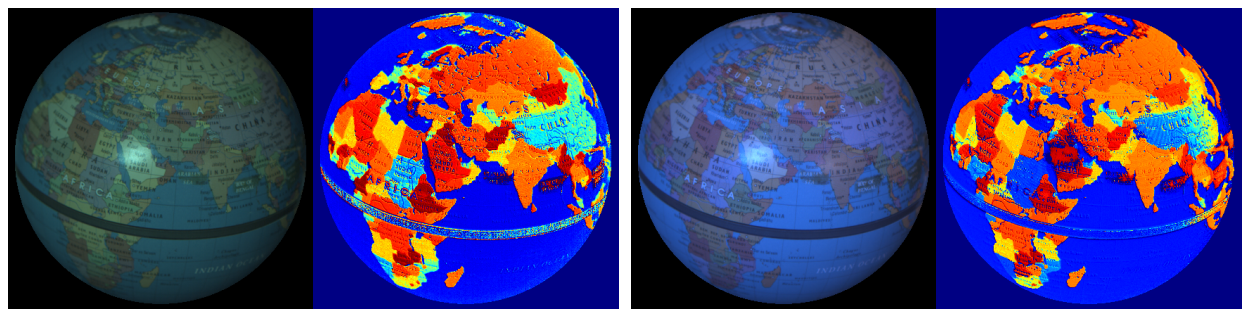

Figure 3: Pseudo-colored generalized hue images, each computed from a single RGB image of a globe under point source illumination having a distinct color. Generalized hue is invariant to both specularities and diffuse shading, and is discriminative only with respect to the spectral reflectance of the surface.

often well-approximated by the Lambertian model, this implies that the specularinvariant image often: 1) satisfies the 'constant-brightness assumption' underlying most stereo and structure-from-motion systems; and 2) provides access to surface normal information through Lambertian-based photometric reconstruction methods such as shape-from-shading and photometric stereo. As a result, by computing these invariants as a pre-processing step, we can successfully apply many Lambertian-based algorithms to a much broader class of specular, non-Lambertian surfaces. Applications are explored in Sect. 5.

\subsection{Generalized Hue}

An additional invariant is created by taking the ratio between specular invariant channels of Eq. 6. The result,

$$
e_{U} / e_{V}=\mathbf{r}_{1}^{\top} \mathbf{d} / \mathbf{r}_{2}^{\top} \mathbf{d},
$$

is independent of both the diffuse and specular geometric scale factors $\sigma_{d}$ and $\sigma_{s}$. As shown in Fig. 1, it is instructive to interpret this ratio as an angle and define

$$
\psi=\tan ^{-1}\left(e_{U} / e_{V}\right)=\tan ^{-1}\left(\mathbf{r}_{1}^{\top} \mathbf{d} / \mathbf{r}_{2}^{\top} \mathbf{d}\right),
$$

which we refer to as generalized hue. Notice that $\psi$ reduces to the standard definition of hue when the source color $\mathbf{s}$ is white.

Examples of generalized hue images are shown in Fig. 3 for a specular globe under two different source colors. In each case, the source vector is measured by imaging a Macbeth color checker, this vector is used to compute a two-channel subspace image as in Eq. 6, and the ratio between the two channels is used to compute $\psi$. Since it depends only on $\mathbf{d}$, the value of $\psi$ within each country on the globe is constant and is invariant to both specular reflections and diffuse shading. 


\section{Color Subspaces}

If we again think of $R G B$ vectors as points in $\mathbb{R}^{3}$, the invariants defined in the previous section are seen to derive from a projection onto the two-dimensional subspace orthogonal to the source vector s. (See left of Fig. 1.) Based on this interpretation, the invariants defined in Eqs. 6 and 7 can be generalized to environments with mixed illumination.

The invariants of the previous section are based on Eq. 1, which in turn is premised on the assumption that the illuminant SPD is constant over the incident hemisphere of a surface point (i.e., that the illuminant 'color' is the same in all directions.) Notationally, if $L\left(\boldsymbol{\omega}_{i}, \lambda\right)$ represents the incident radiance at a surface point, where $\boldsymbol{\omega}_{i}=\left(\theta_{i}, \phi_{i}\right) \in \Omega$ parameterizes the hemisphere of incident directions, the model requires that this input radiance field can be factored (with a slight abuse of notation) as $L(\boldsymbol{\omega}) E(\lambda)$. To relate this to the terms in Eq. 1, recall that $f(\boldsymbol{\theta}, \lambda)$ with $\boldsymbol{\theta}=\left(\theta_{i}, \phi_{i}, \theta_{r}, \phi_{r}\right)$ denotes the BRDF of the surface, and write the image formation equation as

$$
e_{k}=\int_{\lambda} \int_{\Omega} f(\boldsymbol{\theta}, \lambda) L\left(\boldsymbol{\omega}_{i}, \lambda\right) C_{k}(\lambda) \cos \theta_{i} d \boldsymbol{\omega}_{i} d \lambda
$$

According to the dichromatic model, the BRDF of the surface can be decomposed into additive diffuse and specular components, and each of these two components can be factored into a univariate function of wavelength and a multivariate function that depends on the imaging geometry. Finally, assuming a neutral interface, the index of refraction on the surface is constant over the visible spectrum-a valid assumption for many materials - so that the specular function of wavelength is constant. This leads to the common expression for the BRDF of a dichromatic surface,

$$
f(\boldsymbol{\theta}, \lambda)=f_{d}(\boldsymbol{\theta}) R(\lambda)+k_{s} f_{s}(\boldsymbol{\theta}),
$$

where $k_{s}$ is a constant. Substituting into Eq. 9 yields the expressions

$$
\begin{aligned}
\sigma_{d} & =\int_{\Omega} f_{d}(\boldsymbol{\theta}) L\left(\boldsymbol{\omega}_{i}\right) \cos \theta_{i} d \boldsymbol{\omega}_{i} \\
\sigma_{s} & =k_{s} \int_{\Omega} f_{s}(\boldsymbol{\theta}) L\left(\boldsymbol{\omega}_{i}\right) \cos \theta_{i} d \boldsymbol{\omega}_{i} \\
d_{k} & =\int R(\lambda) E(\lambda) C_{k}(\lambda) d \lambda \\
s_{k} & =\int E(\lambda) C_{k}(\lambda) d \lambda .
\end{aligned}
$$


To generalize the model, we consider a mixed-illumination environment whose spectral content can be written in terms of a finite linear basis:

$$
L\left(\boldsymbol{\omega}_{i}, \lambda\right)=\sum_{j=1}^{N} L_{j}\left(\boldsymbol{\omega}_{i}\right) E_{j}(\lambda) .
$$

An example with $N=2$ is an office environment where the illumination in every direction can be described as a mixture of daylight and fluorescent light. When the input radiance field can be decomposed in this manner, the BRDF decomposition of Eq. 10 yields

$$
e_{k}=\sum_{j=1}^{N} \sigma_{d}^{(j)} d_{k}^{(j)}+\sigma_{s}^{(j)} s_{k}^{(j)},
$$

with

$$
\begin{aligned}
\sigma_{d}^{(j)} & =\int_{\Omega} f_{d}(\boldsymbol{\theta}) L_{j}\left(\boldsymbol{\omega}_{i}\right) \cos \theta_{i} d \boldsymbol{\omega}_{i} \\
\sigma_{s}^{(j)} & =k_{s} \int_{\Omega} f_{s}(\boldsymbol{\theta}) L_{j}\left(\boldsymbol{\omega}_{i}\right) \cos \theta_{i} d \boldsymbol{\omega}_{i} \\
d_{k}^{(j)} & =\int R(\lambda) E_{j}(\lambda) C_{k}(\lambda) d \lambda \\
s_{k}^{(j)} & =\int E_{j}(\lambda) C_{k}(\lambda) d \lambda .
\end{aligned}
$$

Equation 12 suggests the existence of a specular invariant that is analogous to the two-dimensional subspace defined of Eq. 6. In that section, the illuminant color is assumed constant over the input hemisphere (which corresponds to $N=1$ in Eq. 12) and the specular invariant subspace computed from a threechannel RGB image is two-dimensional. In general, given an $M$-channel (possibly hyper-spectral) image e and an $N$-dimensional spectral basis $\left\{E_{j}(\lambda)\right\}_{j=1 \ldots N}$ for the incident illumination, there exists a subspace of dimension $(M-N)$ that is independent of all $\sigma_{s}^{(j)}$ and therefore invariant to specular reflections. Letting $\left\{\mathbf{r}_{l}\right\}_{l=1 \ldots(M-N)}$ represent an orthonormal basis for this specular invariant subspace, the $l^{\text {th }}$ component (or 'channel') of the specular invariant image is given by

$$
j_{l}=\mathbf{e}^{\top} \mathbf{r}_{l}=\sum_{j=1}^{N} \sigma_{d}^{(j)} \mathbf{r}_{l}^{\top} \mathbf{d}^{(j)} .
$$

A specular invariant image with $(M-N)$ channels defined by this equation can be treated as an image, and as is the case for the $\mathrm{U}$ and $\mathrm{V}$ channels of Sect. 3, 

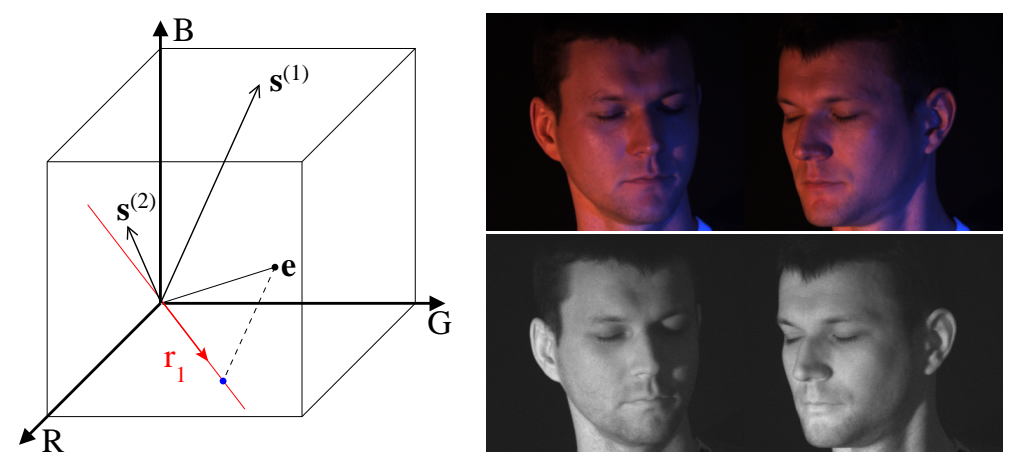

Figure 4: Left: For any mixture of two source SPDs, the specular invariant subspace is one-dimensional. By projecting RGB color vectors onto this line, a specular invariant can still be computed. Right: Two frames of an RGB video of a scene with mixed illumination and the corresponding specular invariants. A blue light on the right and a yellow light on the left induce complex specular effects. Projecting these images onto the one-dimensional subspace orthogonal to the source color vectors in RGB space yields an invariant to specular reflections that preserves diffuse shading information.

the channel values in this image can assume negative values. It is often more convenient to use the monochromatic specular invariant given by

$$
j_{\text {inv }(\mathrm{M}-\mathrm{N})}=\left(\sum_{l=1}^{M-N} j_{l}^{2}\right)^{\frac{1}{2}},
$$

where the subscript $j_{\operatorname{inv}(\mathrm{u})}$ is used to indicate that the grayscale invariant is derived from a $u$-dimensional specular invariant subspace. It is clear that Eqs. 6 and 7 are specific examples of these invariants for the case $M=3$ and $N=1$.

Since the vast majority of cameras record three (RGB) channels, another interesting case to consider is $M=3, N=2$. An example is shown in Fig. 4, where light comes from two sources with different SPDs. These SPDs induce two source color vectors $\mathbf{s}^{(1)}$ and $\mathbf{s}^{(2)}$ in RGB space (these are measured by imaging a calibration target), and by projecting the RGB color vectors of the input image onto the one-dimensional subspace orthogonal to these vectors, we create an image that is void of specular reflection effects.

\subsection{Generalized Hue Under Mixed Illumination}

The concept of generalized hue (Sect. 3.1) can also be extended to handle hyperspectral images and mixed illumination. In an $M$-channel image of a scene illuminated by a mixture of $N$ illuminant SPDs, generalized hue can be defined as a 
scalar function defined on the surface of an $(M-N-1)$-dimensional unit sphere embedded in the $(M-N)$ dimensional diffuse space. The sphere may be parameterized by a vector of angles $\Psi$.

As with RGB sensors and single illuminants, this expanded notion of generalized hue is independent of both shading and specularity, and it is consistent in that it reduces to the standard definition of hue for an RGB image acquired under white light.

\subsection{Practical Considerations}

The quality of the specular invariant signal depends on the spectral characteristics of the scene and the accuracy of the estimated source vectors. We discuss each separately in this section.

\section{Spectral Characteristics}

When a surface is 'white', the spectral reflectance is a constant function of wavelength, so that $R(\lambda)=R$. In this case, since

$$
d_{k}=R \int E(\lambda) C_{k}(\lambda) d \lambda=R s_{k},
$$

it follows that the observed color vector $\mathbf{e}$, the diffuse color vector $\mathbf{d}$ and the source color vector $\mathbf{s}$ are collinear. For these surfaces, the invariant images $\mathbf{j}$ are zero; and as a result, they provide no information about the surface, regardless of the illuminant and sensors that are chosen. This is the same restriction noted by Klinker et al. Klinker et al. (1988); when the diffuse and source colors are the same, there is no way to distinguish between the two reflection components.

More generally, the utility of the proposed invariants relies on the angular separation between the observed color vector $\mathbf{e}$ and the source vectors $\mathbf{s}$. When this separation is small, the signal-to-noise ratio (SNR) in the invariant image can be prohibitively low. This is evident, for example, in the generalized hue image of the globe in the bottom-right of Fig. 3, where the hue variation within the People's Republic of China is seen to be large.

Assuming independent, additive Gaussian noise with zero mean and variance $\sigma^{2}$ in each of the three channels of a color vector $\mathbf{e}_{R G B}$, and assuming $\left\|\mathbf{e}_{R G B}\right\| \leq$ 1 , the signal-to-noise ratio (denoted $\operatorname{SNR}\left(\mathbf{e}_{R G B}\right)$ ) is $10 \log _{10}(1 / \sigma) \mathrm{dB}$. The magnitude of the diffuse color vector $\mathbf{j}$ is related to that of the original color vector by $\|\mathbf{j}\|=\left\|\mathbf{e}_{R G B}\right\| \sin \alpha$, where $\alpha$ is the angle between the source color $\mathbf{s}$ and color vector $\mathbf{e}_{R G B}$ in color space. It follows that

$$
\operatorname{SNR}(\mathbf{j})=\operatorname{SNR}\left(\mathbf{e}_{R G B}\right)+10 \log _{10}(\sin \alpha) .
$$




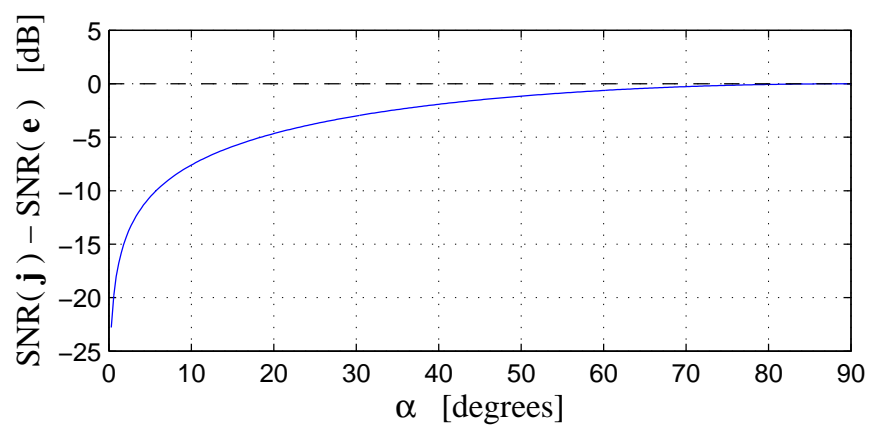

Figure 5: The signal-to-noise ratio (SNR) of the two-channel diffuse image (j) relative to that of the original image (e) as a function of $\alpha$, the angle between $\mathbf{e}$ and the source color $\mathbf{s}$ in the RGB cube.

This relationship is shown in Fig. 5, and it suggests that when the angle between the image and the source color is less than $10^{\circ}$, the two-channel diffuse signal suffers severe degradation.

In practice, this can be improved by increasing the SNR of the input images using multiple exposures (Grossberg and Nayar, 2003). Additionally, since surface points with low SNR can be detected by monitoring the angle between the source colors $\mathbf{s}$ and the input color vectors $\mathbf{e}_{R G B}$, this information can be easily incorporated into any robust vision algorithm (see, e.g., van de Weijer and Gevers, 2004).

\section{Source Color}

It is difficult to make general statements regarding the sensitivity of these invariants to errors in the source color estimates, because this sensitivity depends on the sensitivity functions as well as the spectral reflectances and illuminant SPDs of a particular scene. We can, however, gain some insight from the simple case of a homogeneous surface under a single illuminant. We present a qualitative description of this case here; related quantitative empirical results are presented in Sect. 5.4.2.

RGB observations of a homogeneous surface under a single illuminant lie in the dichromatic plane spanned the source and diffuse vectors s and d. Assuming the source vector is known, a two-channel invariant $\mathbf{j}=\left(j_{1}, j_{2}\right)$ is computed by projecting the vectors onto the subspace orthogonal to this source vector. When the estimate of the source color is inaccurate, the computed invariant also contains error. To describe sensitivity, we consider the square of the grayscale invariant $j_{\text {inv(2) }}^{2}=j_{1}^{2}+j_{2}^{2}$ and compute its derivatives with respect to angular variations in s.

Let $\left\{\mathbf{r}_{1}, \mathbf{r}_{2}\right\}$ be an orthonormal basis for the subspace orthogonal to $\mathbf{s}$, and 
choose this basis such that $\mathbf{r}_{1}$ is in the dichromatic plane. Since the observed color vectors also lie in the dichromatic plane, any one vector will have coordinates of the form $\mathbf{e}=\left(e_{1}, 0, e_{3}\right)$ in the coordinate system defined by $\mathbf{r}_{1}, \mathbf{r}_{2}$, and $\mathbf{r}_{1} \times \mathbf{r}_{2}=\mathbf{s}$. Thus, the squared value of the grayscale invariant is simply $e_{1}^{2}$.

To describe a perturbation of the source direction, we consider a small rotation about an axis (a, say) orthogonal it. The pencil of rotation axes orthogonal to $\mathbf{s}$ can be parameterized by the angle from $\mathbf{r}_{1}$ (so that $\mathbf{a}(\varphi)=(\cos \varphi, \sin \varphi, 0)$ ), and the 'noisy' invariant $\left(j_{\operatorname{inv}(2)}^{2}\right)$ that results from a rotation by angle $\theta$ about any one of these axes is

$$
j_{\text {inv }(2)}^{2}=<\mathbf{R}_{\theta, \varphi} \mathbf{r}_{1}, \mathbf{e}>^{2}+<\mathbf{R}_{\theta, \varphi} \mathbf{r}_{2}, \mathbf{e}>^{2} .
$$

Here, the rotation matrix $\mathbf{R}_{\theta, \varphi}$ is obtained from the axis angle representation as usual,

$$
\mathbf{R}_{\theta, \varphi}=\mathbf{I}+\sin \theta[\mathbf{a}(\varphi)]_{\times}+(1-\cos \theta)[\mathbf{a}(\varphi)]_{\times}^{2},
$$

where $[\cdot]_{\times}$is the skew symmetric matrix equivalent of the cross product.

A measure of the sensitivity of the invariant is obtained by taking the derivative of Eq. 16 with respect to $\theta$ and evaluating it at $\theta=0$ :

$$
\left.\frac{\partial j_{\operatorname{inv}(2)}^{2}}{\partial \theta}\right|_{\theta=0}=2 e_{1} e_{3} \sin \varphi .
$$

This expression reveals that the sensitivity of the invariant is highly asymmetric. When $\varphi=0$, the rotation axis lies in the dichromatic plane, and the source vector is perturbed in a direction orthogonal to that plane. In this case, the derivative is zero and the invariant is largely unaffected by small perturbations of the source estimate. In contrast, when the source color is perturbed within the dichromatic plane (e.g., $\varphi=\pi / 2$ ), the magnitude of the derivative is maximal.

For any perturbation direction (i.e., for any $\varphi$ ), the sensitivity is proportional to the product of the two non-zero components of the color vector e. Thus, if we consider vectors of equal norm, the sensitivity is largest when the angle between the observed color vector $\mathbf{e}$ and the source vector $\mathrm{s}$ is $45^{\circ}$.

\section{Source Color and Interreflections}

In cases of significant interreflection, it is possible for one surface point ( $\mathbf{p}$, say) to specularly reflect light that is first reflected at another point. When the first reflection is diffuse, the reflected spectral radiance is modulated by the spectral reflectance of the surface, and in general, it is not spectrally equivalent to the scene illuminant SPD. Thus, with respect to $\mathbf{p}$ the first point behaves much like a light source having a distinct SPD, and the effective source vector at point $\mathbf{p}$ is different 
from $\mathbf{s}$. In this case, the intensity observed at $\mathbf{p}$ does not follow the image formation model of Eq. 1 (or Eq. 12 in the mixed case), and the proposed invariants may be contaminated by specular effects.

One method for handling interreflection effects is to locally estimate the effective source colors, and to allow these source colors to vary from point to point. As shown by Nayar et al. (1997), this can be accomplished by capturing multiple exposures from a fixed viewpoint with polarization filters at difference orientations. For the purposes of this paper, however, we assume interreflection effects to be negligible so that that effective source vectors are the same at every point and a single image can be used as input.

\section{Applications and Evaluation}

This section investigates the utility of the subspace-based invariants for a number of vision algorithms and compares the results to those obtained using standard grayscale images $e=\left(e_{R}+e_{G}+e_{B}\right) / 3$. For RGB images, when the illumination is a mixture of two known SPDs, the two-channel specular invariant $j_{1}$ from Eq. 13 is grayscale and is equal to $j_{\operatorname{inv}(1)}$ from Eq. 14. On the other hand, a single-SPD specular invariant computed from an RGB image includes two diffuse channels $\left\{j_{1}, j_{2}\right\}$, which can be combined into a grayscale invariant $j_{\text {inv (2) }}$ using Eq. 14. In this case, one can also compute generalized hue, which can be use to replace conventional hue as a material descriptor. The results in this section show that these invariants can have advantages over conventional grayscale and hue images in the presence of specular reflections.

For the experiments in this section, the source colors are measured by imaging a Macbeth color checker in an offline calibration procedure, and we focus on cases in which the diffuse and source color vectors are distinct. A quantitative investigation of the sensitivity with respect to noise in the measured source colors is provided in Sect. 5.4.2.

\subsection{Binocular Stereo}

The vast majority of binocular stereo algorithms are based (either explicitly or implicitly) on the assumption that surfaces are Lambertian. Since specular reflections violate this assumption, stereo reconstructions of specular surfaces are often inaccurate. The most common approach to handle specular effects in binocular stereo is to treat them as outliers. These outliers can be either explicitly detected and removed (Brelstaff and Blake, 1988) or handled implicitly using robust techniques (Yoon and Kweon, 2006b). Instead of treating them as outliers, one may 
also reduce specular reflection effects by modifying the stereo matching function to permit a more general relationship between matched regions (Kim et al., 2003). Alternatively, one can use enhanced acquisition systems that allow the effects of specularities to be reduced or eliminated. Examples include multi-view acquisition schemes (Bhat and Nayar, 1998; Li et al., 2002; Jin et al., 2005), and binocular schemes with active illumination (Zickler et al., 2003; Tu and Mendonça, 2003; Davis et al., 2005b).

More directly related to the present work are reconstructions systems that address specular reflection effects using color information. One approach is to solve the (ill-posed) problem of explicit specular/diffuse separation and use the diffuse images for stereo correspondence. This is explored by Lin et al. (2002), who show that the problem can be more manageable when additional viewpoints are available. Another approach is to use stereo matching based on specular invariants. For the case of monochromatic illumination, binocular stereo using a non-linear specular invariant (which does not preserve diffuse shading information) has been explored by Yoon and Kweon (2006a), and a method that exploits color information in a multi-view system with monochromatic illumination is presented by Yang et al. (2003).

Here we investigate the use of the proposed invariants, which are based on linear transformations and are applicable in both monochromatic and mixed illumination environments. In cases of significant specular reflections and complex illumination conditions, we can improve the accuracy of existing stereo algorithms by computing these specular invariants as a pre-process. Figure 6 compares the results of two binocular stereo algorithms (Birchfield and Tomasi, 1998; Boykov et al., 1998) applied to grayscale $e$ and single-illuminant invariant $j_{\text {inv(2) }}$ images derived from a rectified RGB stereo pair. There is a dramatic improvement in the quality of reconstruction when specular invariant images are used. This point is further emphasized in Fig. 7, which compares binocular stereo results obtained using conventional grayscale images, the single-illuminant (2D subspace) invariant $j_{\text {inv(2) }}$, and the two-color (1D subspace) invariant $j_{\text {inv(1) }}$. In this case, the original RGB image includes two specular highlights caused by blue and yellow illuminants. The blue highlight is largely eliminated in the single-color invariant $j_{\operatorname{inv}(2)}$, while image $j_{\text {inv(1) }}$ is invariant to specular reflections of both colors. As expected, the results from the grayscale and single-color invariant images are poor in specular regions, and the depth map obtained using $j_{\text {inv(1) }}$ is significantly improved.

\subsection{Optical Flow}

Motion estimation through the computation of optical flow is another example of an application that can benefit from specular invariance. Recovering dense opti- 


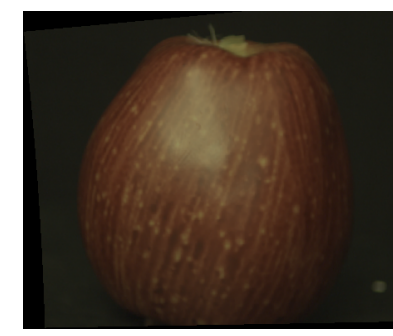

Grayscale
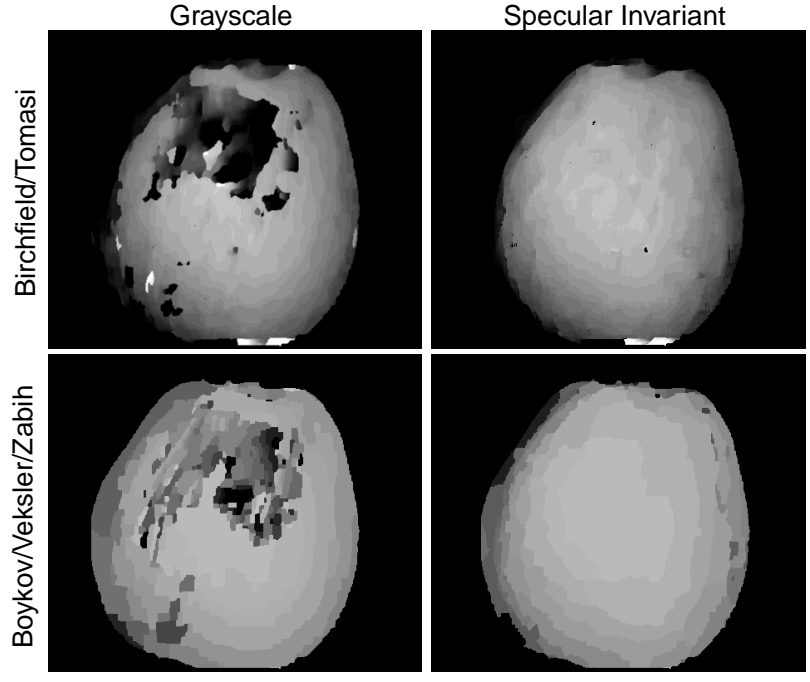

Figure 6: Stereo reconstructions under a single-color illuminant. Both conventional grayscale images and specular invariant images (Eq. 7) are computed from a rectified stereo pair (top) and these are used as input to existing binocular stereo algorithms. Middle row: disparity maps obtained from the grayscale (left) and specular invariant (right) images using the method of Birchfield and Tomasi (1998). Bottom row: those obtained using the method of Boykov et al. (1998).

cal flow relies on the 'constant-brightness assumption', which is violated when an observer moves relative to a static, specular scene.

As is the case with stereo, existing work has shown that color information can be exploited to deal with violations of the constant-brightness assumption (see Barron and Klette (2002) for a survey). Most existing algorithms exploit color by computing either a shading invariant (e.g., normalized RGB) or a white-illuminant specular invariant (e.g., hue) as a pre-process, and studies have shown that these can provide improved estimates of the optical flow field.

We approach the problem of optical flow in a similar spirit using the invariants defined in Sect. 4, which have the advantages of handling non-white illuminants 

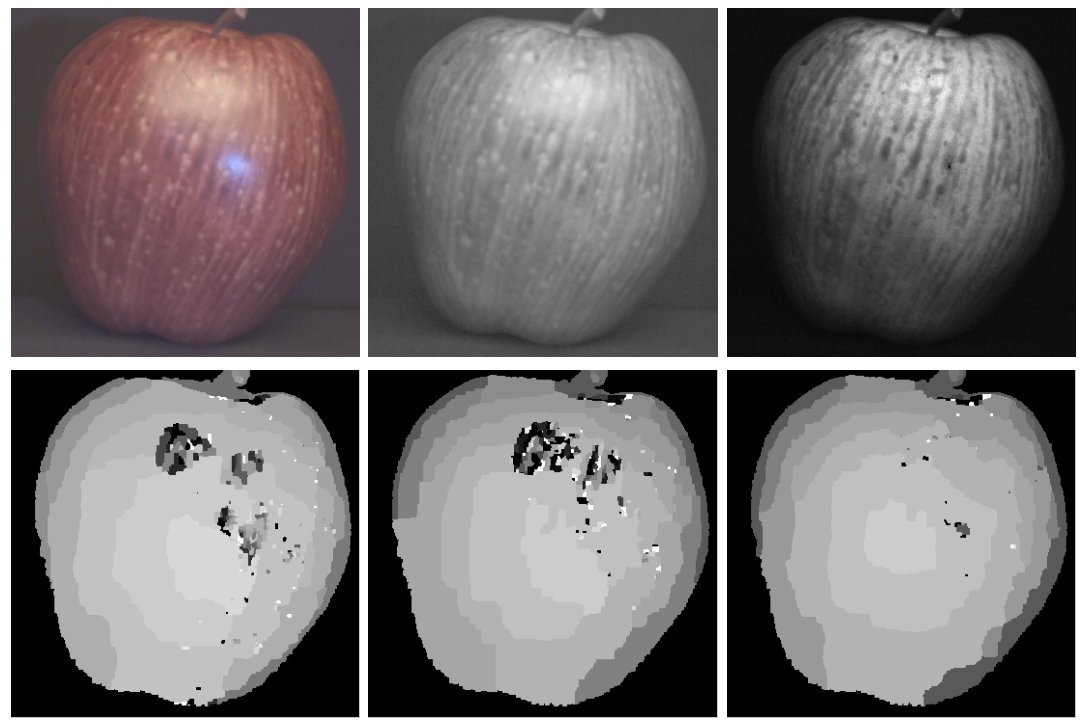

Figure 7: Stereo reconstruction under mixed illumination. Top left: One image of an input stereo pair with blue and yellow illumination. Top center: Single-color invariant image $j_{\operatorname{inv}(2)}$ from Eqs. 13 and 14 with $\mathbf{s}$ in the direction of the blue source. Top right: Two-color invariant $j_{\text {inv(1) }}$ obtained by projecting to the 1D subspace orthogonal to both sources. Bottom row: depth map obtained using the stereo algorithm of Boykov et al. (1998) in each case.

and mixed lighting environments. Figure 8 shows a comparison of optical flow estimation in the presence of specular reflections under a single-color illuminant. An RGB image sequence is captured by a camera translating horizontally relative to a static scene. The sequence is used to compute a conventional grayscale sequence $e(t)$ and a single-color invariant sequence $j_{\operatorname{inv}(2)}(t)$, and these are used as input to a robust optical flow algorithm (Black and Anandan, 1993). Since the camera undergoes pure translation, the 'ground truth' flow lies along parallel horizontal lines. As the figure shows, in regions that are predominantly diffuse, the flow obtained in both cases is close to the ground truth. In regions of specularity, however, there is a significant improvement in the quality of estimated flow when specular invariant images are used.

More interesting is the case of optical flow estimation under mixed illumination, which is shown in Fig. 9. A similar sequence is captured under illumination that is a mixture of two distinct SPDs, and the sequence is used to compute a conventional grayscale sequence $e(t)$, a single-color invariant sequence $j_{\operatorname{inv}(2)}(t)$, and a two-color invariant sequence $j_{\operatorname{inv}(1)}(t)$. These three videos are used as input to the same optical flow algorithm (Black and Anandan, 1993). The left of Fig. 9 


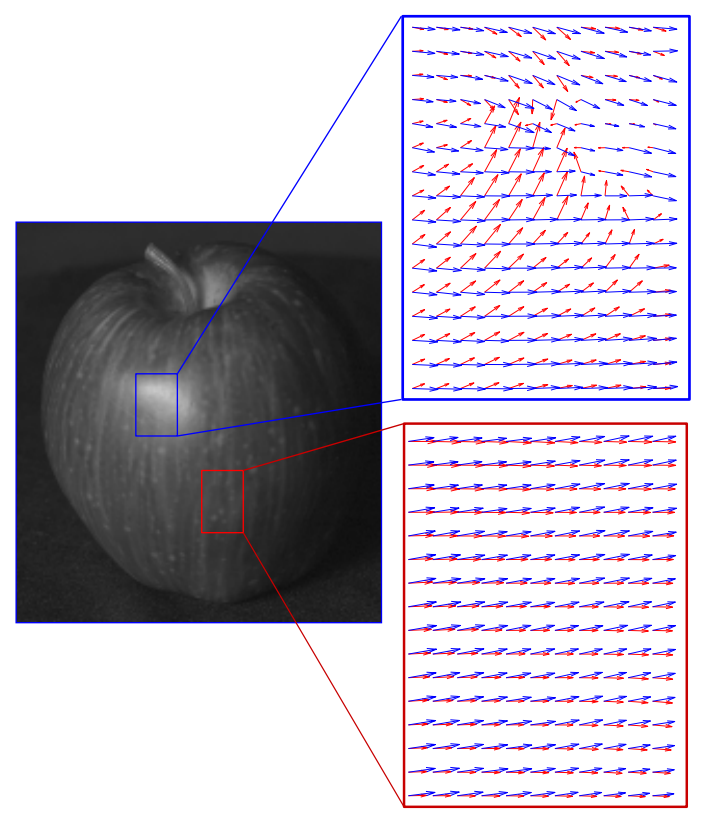

Figure 8: Optical flow under a single-color illuminant. An RGB image sequence is captured by a camera translating left relative to a specular apple. Both conventional grayscale and specular invariant images (Eq. 7) are computed from this RGB sequence, and these are used as input to Black and Anandan's robust optical flow algorithm (Black and Anandan, 1993). Left: Single frame from the grayscale sequence. Right: flows obtained for regions that are highly specular and predominantly diffuse. Red flow is computed from the grayscale sequence and is severely corrupted by specular reflection. Blue flow is computed from the specular invariant sequence and is much closer to ground truth, which is horizontal and to the right.

shows a single image from each sequence, and the right shows the recovered flows in the indicated window. The flow recovered using the conventional grayscale and single-color invariant sequences are severely corrupted by specular highlights. In contrast, the flow computed from the mixed-illuminant invariant (shown in red) is close to the ground truth and is largely unaffected by these non-Lambertian effects.

\subsection{Shape from Shading}

The previous two sections demonstrate the utility of the specular invariant for stereo matching and optical flow, both of which benefit from the fact that the specular invariant images do not change with viewpoint. The next three sections show 


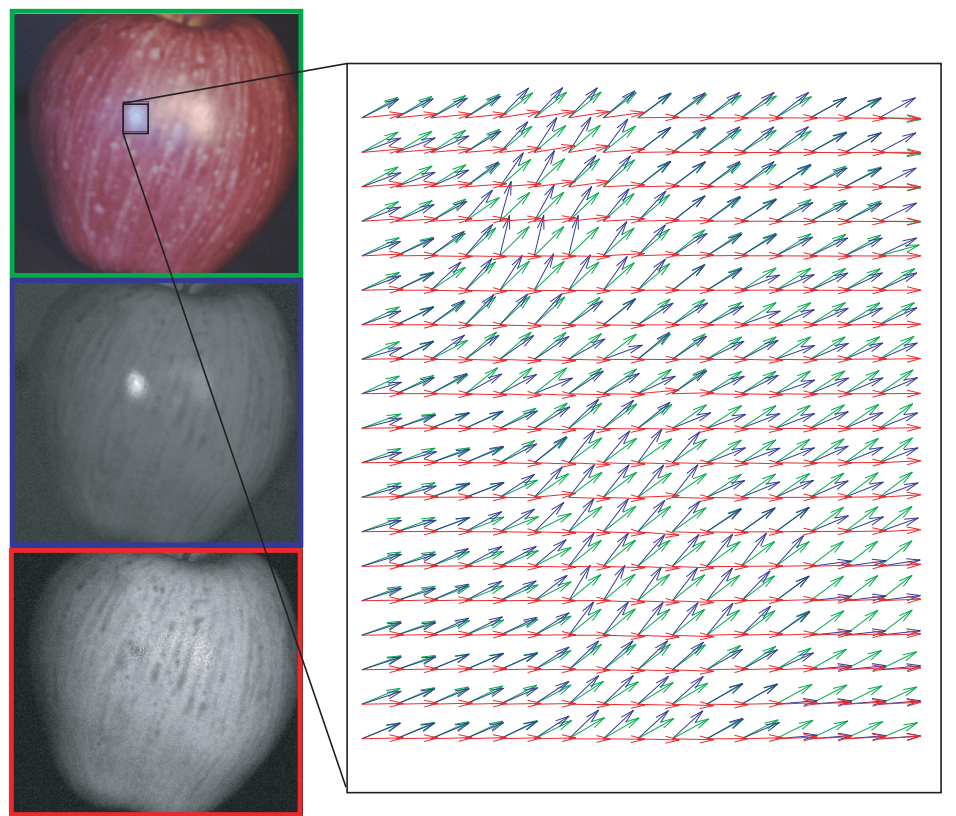

Figure 9: Optical flow under mixed illumination. An RGB image sequence (top left) is captured by a camera translating left relative to a specular apple under yellow and blue illumination. Derived conventional grayscale $e(t)$, yellow-invariant $j_{\operatorname{inv}(2)}(t)$ (left middle), and two-color invariant $j_{\text {inv(1) }}(t)$ (left bottom) sequences are computed and used as input to Black and Anandan's robust optical flow algorithm (Black and Anandan, 1993). Right: flows obtained in the three cases. Green and blue flows are from grayscale and yellowinvariant sequences, respectively, and both are corrupted by specular reflections. Red flow is computed from the two-color invariant and is much closer to ground truth, which is horizontal and to the right.

that since they preserve diffuse (ideally Lambertian) shading information, these invariants can also be used to enhance photometric reconstruction methods.

In shape from shading, one seeks to recover surface shape from the photometric information available in a single image. The vast majority of the existing methods assume Lambertian reflectance, and even then the problem is a difficult one. Of the small number of methods that consider non-Lambertian effects, most assume reflectance to be of a specific parametric form-such as the Torrance-Sparrow or Oren-Nayar models - which must be known a priori (Ahmed and Farag, 2006; Bakshi and Yang, 1994; Ragheb and Hancock, 2001). The use of color in shape from shading is rare. One notable example is the work of Tian and Tsui (1997), which considers reflectance that is a linear combination of a Lambertian diffuse 


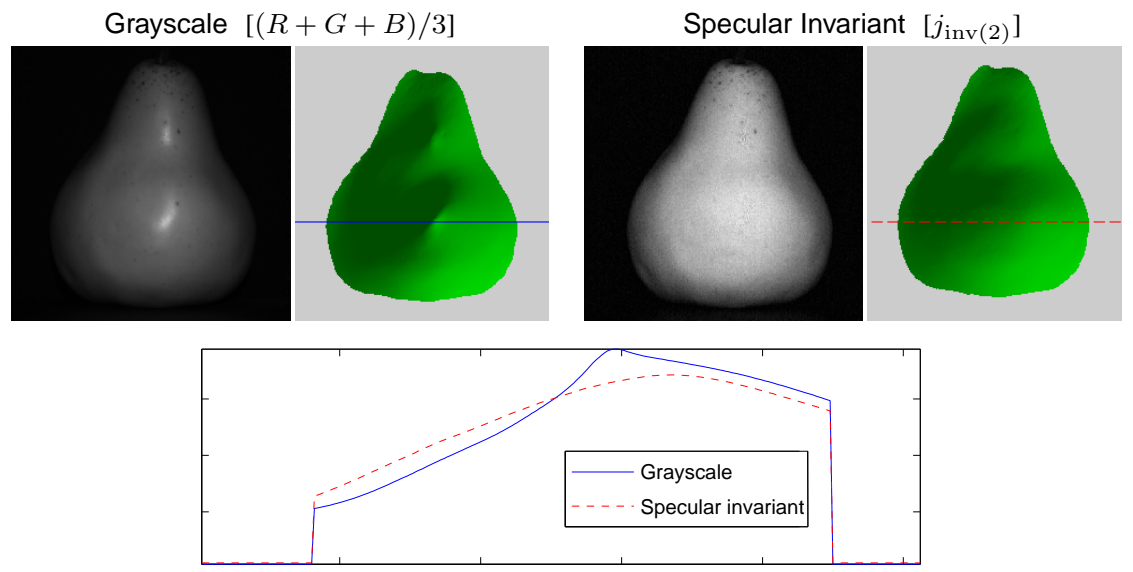

Figure 10: Shape from shading comparison. Top: An RGB image of a pear is used to compute conventional grayscale (left) and specular invariant (right) images, and these are input to a shape from shading algorithm (Zheng and Chellappa, 1991) yielding the surfaces shown in green. Bottom row: cross-sections of the recovered surfaces along the indicated horizontal lines.

component and an ideal specular spike.

The invariants presented in Sect. 4 provide a means for considering a much broader class of surfaces. By combining these invariants with existing Lambertianbased methods for shape from shading, one can recover shape for surfaces having rather arbitrary specular components (i.e., general $f_{s}(\boldsymbol{\theta})$ in Eq. 10) which need not be well-represented by any known parametric form. All that is required is that the surface conforms to the dichromatic model.

When illumination can be described as a single point source in direction $\mathbf{l}$ (say) and the diffuse reflectance at a surface point is Lambertian, we can write $\sigma_{d}=$ $f_{d} \mathbf{n}^{\top} \mathbf{l}$, where $\mathbf{n}$ is the surface normal at the point and $f_{d}$ is the albedo. When this is true, the specular invariant image of Eq. 14 reduces to

$$
j_{\mathrm{inv}(2)}=f_{d}\left(\left(\mathbf{r}_{1}^{\top} \mathbf{d}\right)^{2}+\left(\mathbf{r}_{2}^{\top} \mathbf{d}\right)^{2}\right)^{\frac{1}{2}} \mathbf{n}^{\top} \mathbf{l}
$$

which is the image formation equation for a Lambertian surface with an effective albedo given by the first two terms. Thus, the specular invariant can be used directly as input to any Lambertian-based shape from shading algorithm.

The benefit of this approach is demonstrated in Fig. 10, where we assess the performance of a conventional shape from shading algorithm (Zheng and Chellappa, 1991) for both a grayscale image $e$ and a single-SPD invariant image $j_{\text {inv(2) }}$. 
The top of the figure shows grayscale and specular invariant images computed from an RGB image of a pear, and the middle row shows the surfaces that are recovered by applying the same algorithm in the two cases. The solid blue profile in the bottom graph shows that specular reflections cause severe artifacts when the algorithm is applied to the grayscale image. In contrast, as shown by the dashed red profile, one can obtain improved results using the same algorithm by computing the specular invariant as a pre-processing step.

\subsection{Photometric Stereo}

In photometric stereo, one seeks to recover shape from a set of images acquired from a fixed viewpoint under multiple illumination conditions. Like shape from shading, photometric stereo requires the inversion of the image formation process, and as a result, existing methods also require significant knowledge about the reflectance of surfaces in a scene. Many photometric stereo techniques assume that surfaces are Lambertian (Woodham, 1978), and others assume the reflectance to be given a priori by a reference object (Silver, 1980), a linear basis of reference objects (Hertzmann and Seitz, 2003), or by a parametric BRDF model (Ikeuchi, 1981; Nayar et al., 1990; Tagare and deFigueiredo, 1991). When these reflectance assumptions are not satisfied, the accuracy of the recovered shape can be compromised.

Coleman and Jain (1982) were perhaps the first to present a photometric technique for reconstructing non-Lambertian surfaces without an explicit reflectance model. In their method, the BRDF is assumed to be a linear combination of a Lambertian diffuse component and an undefined specular component with limited angular support. When four point-source illuminations are available, specular measurements can be treated as outliers and discarded, provided that the illumination directions are far from one another relative to the angular extent of the specular lobe. (This ensures that the specular reflectance component is zero for three of the four observations of each surface point.) Barsky and Petrou (2003) refine this technique by using color information to improve the detection of specular measurements. Like the original work, however, specular measurements are treated as outliers, and the specular component is assumed to have limited angular support.

Another approach to photometric stereo for non-Lambertian surfaces is to assume dichromatic surfaces, and to explicitly separate the diffuse and specular components as a pre-processing step. This is the approach taken by Schlüns and Wittig (1993), who assume homogeneous dichromatic surfaces, and separate the diffuse and specular components using color histogram analysis techniques similar to Klinker et al. (1988). Sato and Ikeutchi (1994) take a similar approach, but avoid the restriction to homogeneous surfaces by using a large number of light 
source directions to compute a distinct color histogram at each point. Because these methods explicitly recover the diffuse and specular components, they have the additional benefit of providing an estimate of the diffuse color $\mathbf{d}$ at each point in addition to recovering the surface shape. Since they are based on explicit specular/diffuse separation, however, they are subject to the restrictions discussed in Sect. 2. Most importantly, they assume that the specular lobe is narrow relative to the surface curvature, an assumption similar to that underlying the four-source method of Coleman and Jain (1982).

By using the invariants from Sect. 4 in conjunction with existing Lambertianbased methods for photometric stereo, many of these limitations can be overcome. In fact, this provides a reconstruction method that operates completely independent of specular reflections (i.e., independent of $f_{s}(\boldsymbol{\theta})$ in Eq. 10) and therefore requires no additional assumptions regarding the specular behavior of a surface. In this sense, this approach to photometric stereo is related to other recent reconstruction methods that exploit physical properties such as reflectance isotropy ( $\mathrm{Lu}$ and Little, 1999), reciprocity (Magda et al., 2001; Zickler et al., 2002), the constancy of radiance in free space (Magda et al., 2001; Koudelka et al., 2001), and light transport constancy (Davis et al., 2005a) to enable accurate reconstructions of very broad classes of surfaces. An important difference, however, is that the photometric stereo method described here requires a simple acquisition system and is quite easy to implement.

To use the proposed invariants for photometric stereo, we assume directional monochromatic illumination as in the previous section. Let $\mathbf{j}^{1}, \mathbf{j}^{2}, \mathbf{j}^{3}$ be three twochannel color vectors produced by observing a single point under three different lighting directions $\mathbf{l}^{1}, \mathbf{l}^{2}, \mathbf{l}^{3}$. specular invariants are computed from the RGB images according to Eq. 13. Assuming Lambertian diffuse reflectance, we see that

$$
\mathbf{j}^{k}=\left[j_{1}^{k}, j_{2}^{k}\right]^{\top}=\left(\mathbf{n}^{\top} \mathbf{l}^{k}\right) \boldsymbol{\rho}
$$

with

$$
\boldsymbol{\rho}=\left[\rho_{1}, \rho_{2}\right]^{\top}=f_{d}\left[\mathbf{r}_{1}^{\top} \mathbf{d}, \mathbf{r}_{2}^{\top} \mathbf{d}\right]^{\top}
$$

being an effective two-channel albedo, and it follows that these specular invariant images can be used as input to a Lambertian photometric stereo algorithm. In what follows, we adapt the algorithm of Barsky and Petrou (2001) that was originally designed to handle RGB images of Lambertian scenes.

Similar to Barsky and Petrou (2001), a shading vector is defined as $\mathbf{h}=$ $\left[h^{1}, h^{2}, h^{3}\right]^{\top}=\left[\begin{array}{lll}\mathbf{l}^{1} & \mathbf{l}^{2} & \mathbf{l}^{3}\end{array}\right]^{\top} \mathbf{n}$, and the invariant images resulting from the three 

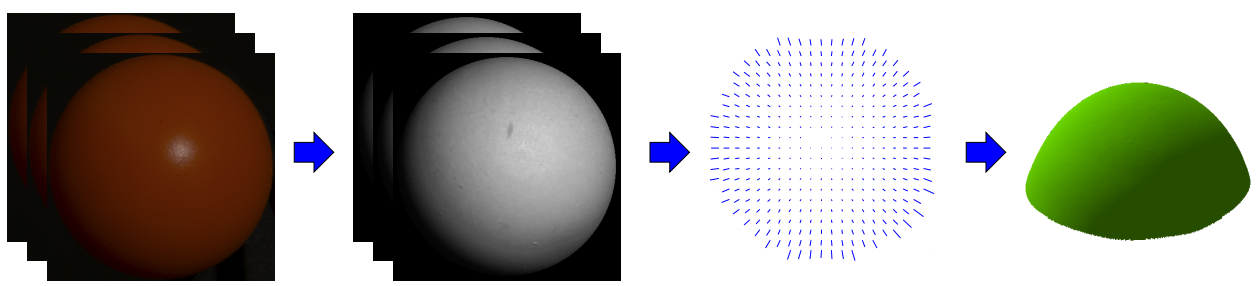

Figure 11: Photometric stereo procedure. Three or more RGB images are acquired under known illumination conditions, and specular invariants $\mathbf{j}$ are computed according to Eq. 13. The invariants represent diffuse images of the object, and these are used with standard photometric stereo techniques to estimate the surface normal at each pixel. The normals are integrated to recover the surface.

lighting directions are combined in an intensity matrix satisfying

$$
\mathbf{J}=\left[\begin{array}{cc}
j_{1}^{1} & j_{2}^{1} \\
j_{1}^{2} & j_{2}^{2} \\
j_{1}^{3} & j_{2}^{3}
\end{array}\right]=\left[\begin{array}{ll}
h^{1} \rho_{1} & h^{1} \rho_{2} \\
h^{2} \rho_{1} & h^{2} \rho_{2} \\
h^{3} \rho_{1} & h^{3} \rho_{2}
\end{array}\right]=\mathbf{h} \boldsymbol{\rho}^{\top} .
$$

The least-squares estimate of the shading vector $\mathbf{h}$ is computed from the intensity matrix; it is the principal eigenvector of $\mathbf{J J}^{\top}$. Once the shading vector is determined, the surface normal is found by solving the matrix equation $\mathbf{h}=\left[\begin{array}{lll}\mathbf{l}^{1} & \mathbf{l}^{2} & \mathbf{l}^{3}\end{array}\right]^{\top} \mathbf{n}$. This reconstruction procedure is outlined in Fig. 11, and it can be applied without change to any number of images larger than three.

\subsubsection{Experimental Results}

Photometric stereo provides a convenient means for quantitative analysis of the proposed invariant, since we can directly measure the accuracy of reconstructed shapes having different material properties. To perform such an analysis, we painted five identical spheres, shown in Fig. 12, with standard latex paints that were mixed to have approximately the same color pigment and five different levels of glossy finish: flat, eggshell, satin, semi-gloss, and high-gloss. The observed incidentplane BRDFs of these spheres are shown in Fig. 13.

For each sphere, a set of four high dynamic range (HDR) images were captured from a fixed viewpoint and four known illumination directions. The source color was calibrated by imaging a Macbeth color checker, and it was used to compute the specular invariants $\mathbf{j}$ and $j_{i n v(2)}$ according to Eqs. 13 and 14. The second column of Fig. 12 confirms that the specular invariant $j_{i n v(2)}$ depends largely on the diffuse reflectance. 


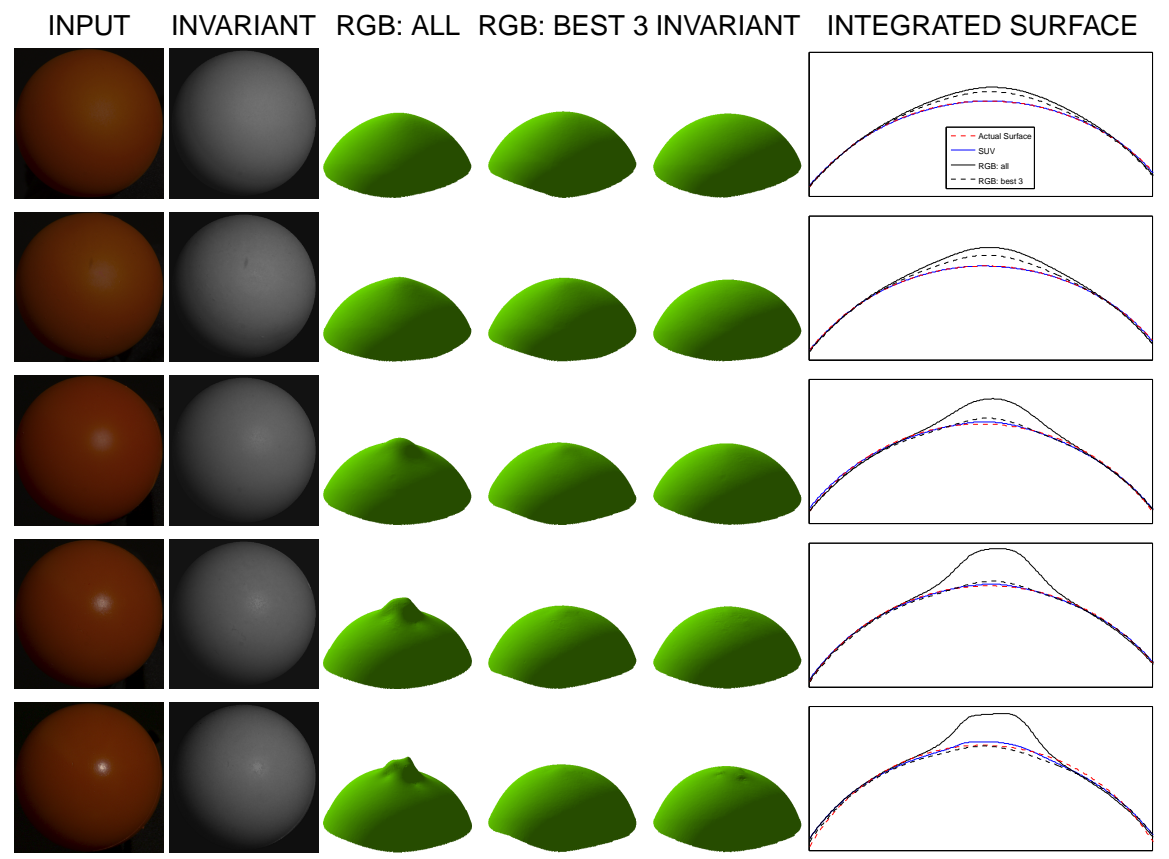

Figure 12: Comparison of photometric stereo methods. Five red spheres with increasing specular reflectance are each observed under four illumination directions, and these images are used to recover the surface. From left to right, each row shows: i) an input RGB image, ii) the corresponding specular invariant $\mathbf{j}$ from Eq. 13, iii) surfaces integrated from the surface normals estimated by three photometric stereo methods, and iv) cross-sections of the surfaces overlaid on the true shape.

Using the two-channel specular invariant images, the surface normals of each sphere were estimated using the photometric stereo method described above. As a means of comparison, we implemented two alternative RGB-based photometric techniques. The first method uses all four RGB images and assumes Lambertian reflectance (Barsky and Petrou, 2001). The second method assumes Lambertian+specular reflectance and reconstructs the surface by choosing the three 'least specular' RGB measurements at each pixel (Barsky and Petrou, 2003; Coleman and Jain, 1982). The results are shown in Figs. 12 and 13. The recovered surfaces, including cross-sections overlaid on the true shape, are displayed in Fig. 12. Quantitative results are shown in Fig. 13, with the bottom of that figure displaying the angular difference between the true and estimated surface normals as a function of increasing specularity. These results demonstrate that the invariantbased reconstruction is largely independent of the specular reflectance, whereas both the four-image and three-image RGB methods are affected by it. The four- 

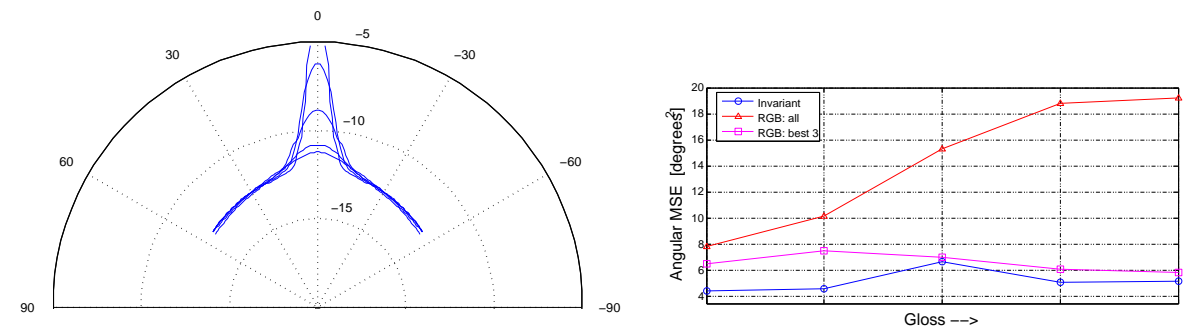

Figure 13: Comparison of photometric stereo methods. Left: Relative BRDFs (in decibels) of the five red spheres of Fig. 12 as a function of half-angle. Right: Mean-square angular error in the recovered surface normals as a function of increasing specularity using both the proposed specular invariants and existing RGB methods.

image method (Barsky and Petrou, 2001) assumes Lambertian reflectance and its performance degrades monotonically as gloss increases; and while the three-image RGB method (Barsky and Petrou, 2003; Coleman and Jain, 1982) performs well for the high-gloss (narrow specular lobe) spheres, it performs less well when the angular support of the specular lobe is large relative to the separation of the light source directions.

Figure 14 shows the results of applying the invariant-based photometric stereo method to two natural objects (a pear and a pumpkin.) Since the computation of the specular invariant is purely local, the method requires no spatial coherence in the image, and it performs well for surfaces with arbitrary texture. This is not true for alternative photometric stereo techniques that rely on explicit diffuse/specular separation (e.g., (Schlüns and Wittig, 1993)), since these methods generally require some form spatial coherence in the spectral reflectance of a surface.

\subsubsection{Sensitivity to Illuminant Color}

Photometric stereo also provides an opportunity to quantitatively evaluate the sensitivity of the proposed invariants to perturbations in the measured illuminant color. This compliments the qualitative analysis presented in Sect. 4.2. To measure sensitivity, we repeated the photometric reconstruction procedure in Fig. 13 using invariants computed with perturbed source vectors. When the source vector is perturbed from its true value, the specular invariant images are contaminated by specular effects, and the reconstruction error in the Lambertian-based photometric stereo result is expected to increase.

Figure 15 shows the result of this experiment using the red sphere from the second row of Fig. 12. Depicted is the angular mean-square error (in degrees) resulting from perturbations of the unit source vector. Since source vectors are are 

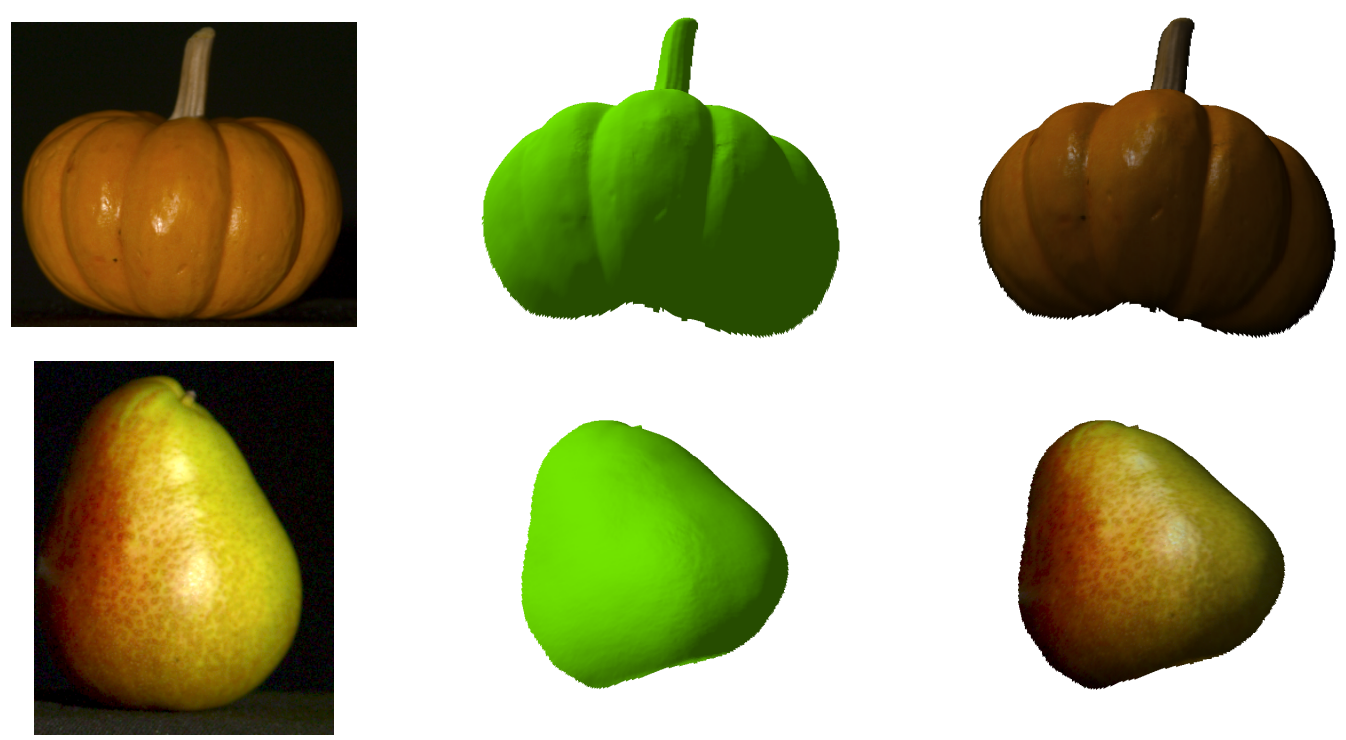

Figure 14: Invariant-based photometric stereo applied to natural surfaces. Left: Input RGB images show significant specular reflectance and texture. By computing the specular invariant, the specular effects are removed, enabling accurate recovery of shape. Middle, Right: Surfaces recovered by integrating the estimated surface normals.

of unit length, the domain of the error function is the unit sphere, and the figure shows the stereographic projection of this error function centered at the true source color (indicated by + ). Concentric circles in Fig. 15 correspond to angular source perturbations of $5^{\circ}, 10^{\circ}$ and $15^{\circ}$, and the diagonal black line is the projection of the dichromatic plane, which is the plane spanned by the diffuse vector of the homogeneous surface and the true source vector.

The qualitative analysis from Sect. 4.2 reveals that the specular invariant is more sensitive to source perturbations within the dichromatic plane than it is to perturbations away from the plane. This effect is also observed in Fig. 15, where a $10^{\circ}$ perturbation within the plane causes the error to increase by nearly a factor of two, while the same angular perturbation in the orthogonal direction induces only a $25 \%$ increase.

While this experiment provides some insight into the sensitivity of the proposed invariants, one must be cautious about the conclusions one draws. Since photometric stereo is an active illumination technique, one typically has the opportunity to directly measure the source color. When this is the case, the noise in the source estimate will be much smaller than the $15^{\circ}$ error considered here. For other applications (stereo, optical flow, etc.) in which the source color is difficult to 


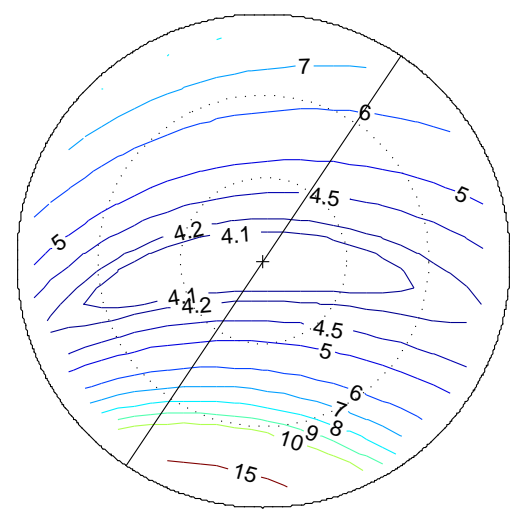

Figure 15: Sensitivity of a photometric stereo reconstruction with respect to errors in estimated source color. The field of surface normals of a sphere from Fig. 12 is recovered using invariant-based photometric stereo with source vectors s perturbed from truth, and the angular MSE in the normal field is recorded. Shown is a contour plot of the stereographic projection of this error (in degrees) as a function of the angular perturbation to the source vector. Concentric circles are cones of source vectors displaced by $5^{\circ}, 10^{\circ}$ and $15^{\circ}$ from the true vector $(+)$, and the diagonal line is the projection of the dichromatic plane for this homogeneous surface. The angular MSE for the true source vector is $3.98^{\circ}$. The reconstruction is more sensitive to source perturbations within the dichromatic plane than those orthogonal to it.

measure or is time-varying, one would need to rely on existing image-based methods for illuminant estimation as discussed in Sect. 2. Angular source errors may be larger in this case-some empirical studies suggest that errors of $10^{\circ}$ are not uncommon Barnard et al. (2002b,a) - and these errors will depend very strongly on the particular materials and illuminants that are present in the scene, the sensitivity functions of the camera being used, and the color constancy algorithm(s) being employed.

\subsection{Photometric/Geometric Reconstruction}

In addition to the applications presented thus far, the specular invariant can be used to improve the performance of a broad class of Lambertian-based reconstruction systems in the presence of specular, non-Lambertian surfaces. This includes methods that combine both geometric and photometric constraints to obtain accurate surface shape (Jin et al., 2004; Lim et al., 2005; Zhang et al., 2003). To provide an example, we use the passive photometric stereo algorithm described by Lim et al. (2005). This method begins with an approximate, piece-wise planar reconstruction 

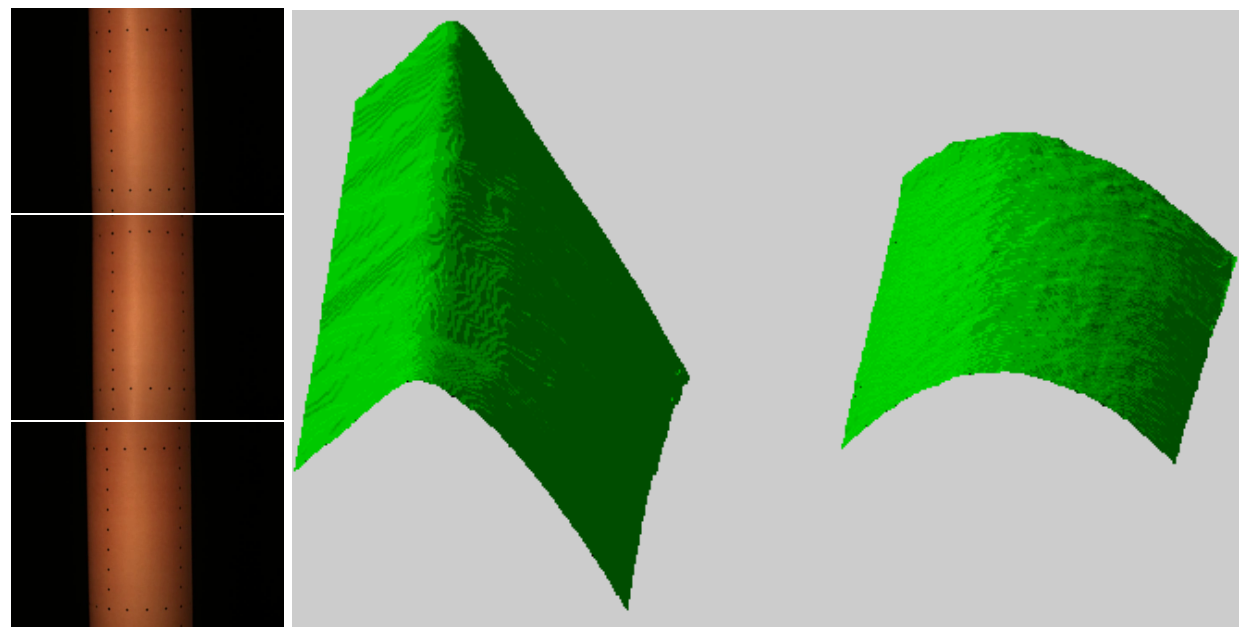

Figure 16: Comparison of shape from combined photometric and geometric constraints. Left: three RGB frames of a specular cylinder moving under fixed view and illumination. Right frame: result of simultaneous tracking and photometric reconstruction (Lim et al., 2005) using both the conventional grayscale (left) and specular invariant (right) sequences.

obtained by tracking a small number of features across a video sequence under (possibly varying) directional illumination. Then, an iterative method based on uncalibrated Lambertian photometric stereo simultaneously refines the reconstruction and estimates the unknown illumination directions.

Figure 16 compares the results obtained from an image sequence that consists of a moderately specular cylinder moving under fixed illumination and viewpoint. The shape is estimated by applying the same algorithm to both the conventional grayscale sequence $(e(t))$ and the specular invariant sequence $\left(j_{\operatorname{inv}(2)}\right)$ computed from the same RGB data. The right-most surface in Fig. 16 shows that the reconstruction obtained using the specular invariant is nearly cylindrical, while that computed from the conventional grayscale sequence is severely corrupted by specular reflections.

\subsection{Material-based Segmentation}

Sections 5.1-5.5 demonstrate the utility of the proposed specular invariants for a variety of visual tasks. This section demonstrates an applications of the second invariant, generalized hue, which is independent of both the specular reflections and diffuse shading in an image. We consider its application to the problem of material-based segmentation, although other potential applications include lighting-insensitive tracking and recognition. 


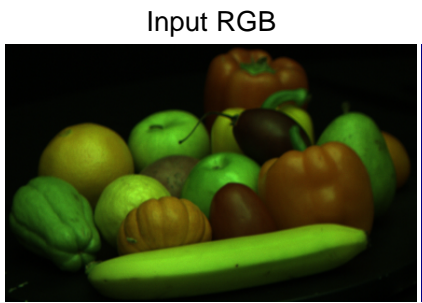

Conventional Hue

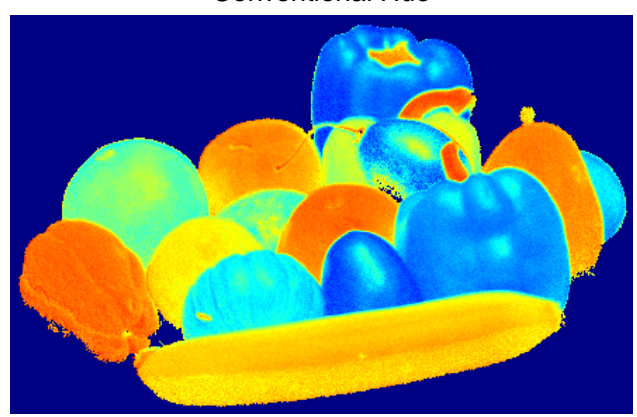

Conventional Grayscale
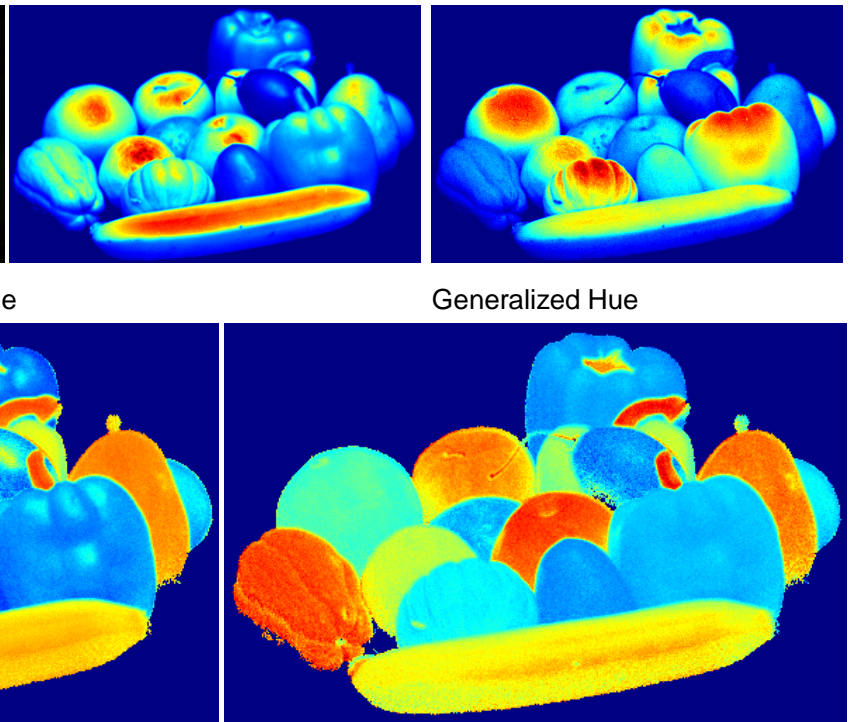

Figure 17: Generalized hue for material-based segmentation. Each panel shows a pseudocolored representation that is computed from the RGB image on the top-left. The generalized hue image on the bottom-right is useful for segmentation because it depends only on the spectral reflectance of the surfaces. The same is not true for a conventional hue image (bottom-left) unless the illuminant is white.

Figure 17 shows an RGB image of a dichromatic scene under uniform source color $(N=1)$ along with a series of pseudo-colored representations related to the invariants presented in Sect. 4. The top row shows conventional grayscale and specular invariant images, and in the latter, the specular effects (most notably on the green apples, the pumpkin, and the red pepper) are largely eliminated. The bottomright of Fig. 17 shows the generalized hue image given by Eq. 8, which is invariant to diffuse shading in addition to specular reflections, and therefore depends only on the spectral reflectance. The fact that the generalized hue within each region is relatively constant suggests that it is a useful representation for segmentation. The same is not true for the conventional hue image (shown on the bottom-left) because the illuminant is not white.

\section{Conclusion}

This paper presents photometric invariants that are derived from color subspaces. They can be efficiently computed from a single image of a dichromatic scene and 
can be applied in cases of both monochromatic and mixed illumination environments. Two important features of these invariants are that: 1) they are free of specular reflectance effects; and 2) they preserve the diffuse shading information in an image. The latter means that they can be used directly for Lambertian-based photometric analysis including shape from shading and photometric stereo.

The invariants are computed point-wise and therefore place no restriction on scene texture. Additionally, while they require knowledge of the effective source color(s), they place no restrictions on the angular distribution of incident light.

The utility of these invariants is demonstrated by their ability to improve the performance of a wide variety of vision algorithms, including those for binocular stereo, motion estimation, and photometric reconstruction. They are directly applicable in cases where the source color is measured or known, and in these cases, they are shown to allow many Lambertian-based algorithms to be applied more successfully to a much broader class of surfaces.

An important next step is to explore applications in uncontrolled environments, where illumination spectra cannot be measured or are time varying. By combining the proposed invariants with existing methods for illuminant estimation and robust Lambertian-based vision algorithms, they may prove to be useful in these cases as well.

\section{References}

Ahmed, A. and Farag, A. (2006). A New Formulation for Shape from Shading for Non-Lambertian Surfaces. Proc. IEEE Conf. Computer Vision and Pattern Recognition, pages II: 1817-1824.

Bakshi, S. and Yang, Y.-H. (1994). Shape from shading for non-lambertian surfaces. In Proc. IEEE Int. Conf. Image Processing, volume 2, pages 130-134.

Barnard, K., Cardei, V., and Funt, B. (2002a). A comparison of computational color constancy algorithms. I: Methodology and experiments with synthesized data. IEEE Trans. Pattern Analysis and Machine Intelligence, 11(9).

Barnard, K., Martin, L., Coath, A., and Funt, B. (2002b). A comparison of computational color constancy algorithms. II. Experiments with image data. IEEE Trans. Image Processing, 11(9):985-996.

Barron, J. L. and Klette, R. (2002). Quantitative color optical flow. In Proc. International Conference on Pattern Recognition, volume 4, pages 251-255, Washington, DC, USA. IEEE Computer Society. 
Barsky, S. and Petrou, M. (2001). Colour photometric stereo: Simultaneous reconstruction of local gradient and colour of rough textured surfaces. In Proc. IEEE Int. Conf. Computer Vision, pages 600-605.

Barsky, S. and Petrou, M. (2003). The 4-source photometric stereo technique for three-dimensional surfaces in the presence of highlights and shadows. IEEE Trans. Pattern Analysis and Machine Intelligence, 25(10):1239-1252.

Bhat, D. and Nayar, S. (1998). Stereo and specular reflection. Int. Journal of Computer Vision, 26(2):91-106.

Birchfield, S. and Tomasi, C. (1998). Depth discontinuities by pixel-to-pixel stereo. In Proc. IEEE Int. Conf. Computer Vision, pages 1073-1080.

Black, M. J. and Anandan, P. (1993). A framework for the robust estimation of optical flow. In Proc. IEEE Int. Conf. Computer Vision, pages 231-236.

Blanz, V. and Vetter, T. (2003). Face recognition based on fitting a 3D morphable model. IEEE Trans. Pattern Analysis and Machine Intelligence, 25(9).

Boykov, Y., Veksler, O., and Zabih, R. (1998). Markov random fields with efficient approximations. In Proc. IEEE Conf. Computer Vision and Pattern Recognition, pages 648-655.

Brainard, D. H. and Freeman, W. T. (1997). Bayesian color constancy. J. Optical Society of America A, 14:1393-1411.

Brelstaff, G. and Blake, A. (1988). Detecting specular reflection using lambertian constraints. In Proc. IEEE Int. Conf. Computer Vision, pages 297-302.

Coleman, E. and Jain, R. (1982). Obtaining 3-dimensional shape of textured and specular surfaces using four-source photometry. Computer Vision, Graphics and Image Processing, 18(4):309-328.

Davis, J., Yang, R., and Wang, L. (2005a). BRDF Invariant Stereo Using Light Transport Constancy. Proc. IEEE Int. Conf. Computer Vision, 1.

Davis, J. E., Yang, R., and Wang, L. (2005b). Brdf invariant stereo using light transport constancy. In ICCV '05: Proceedings of the Tenth IEEE International Conference on Computer Vision (ICCV'05) Volume 1, pages 436-443, Washington, DC, USA. IEEE Computer Society.

Finlayson, G. and Schaefer, G. (2001). Constrained dichromatic colour constancy. In Proc. European Conf. Computer Vision, volume 1, pages 342-358. 
Finlayson, G. D. (1996). Color in perspective. IEEE Trans. Pattern Analysis and Machine Intelligence, 18:1034-1036.

Finlayson, G. D., Hordley, S. D., and Hubel, P. M. (2001). Color by correlation: a simple, unifying framework for color constancy. IEEE Trans. Pattern Analysis and Machine Intelligence, 23:1209-1221.

Gershon, R. (1987). The use of color in computational vision. $\mathrm{PhD}$ thesis, University of Toronto.

Grossberg, M. D. and Nayar, S. K. (2003). High dynamic range from multiple images: Which exposures to combine? In Proc. IEEE Workshop on Color and Photometric Methods in Computer Vision (CPMCV).

Healey, G. (1989). Using color for geometry-insensitive segmentation. J. Optical Society of America A, 6(6):920-937.

Hertzmann, A. and Seitz, S. (2003). Shape and material by example: a photometric stereo approach. In Proc. IEEE Conf. Computer Vision and Pattern Recognition.

Hordley, S. and Finlayson, G. (2006). Reevaluation of color constancy algorithm performance. J. Optical Society of America A, 23(5):1008-1020.

Hordley, S. D., Finlayson, G. D., and Drew, M. S. (2002). Removing shadows from images. In Proc. European Conf. Computer Vision, pages 823-836.

Ikeuchi, K. (1981). Determining surface orientations of specular surfaces by using the photometric stereo method. IEEE Trans. Pattern Analysis and Machine Intelligence, 3(6):661-669.

Jin, H., Cremers, D., Yezzi, A., and Soatto, S. (2004). Shedding light on stereoscopic segmentation. In Proc. IEEE Conf. Computer Vision and Pattern Recognition.

Jin, H., Soatto, S., and Yezzi, A. J. (2005). Multi-view stereo reconstruction of dense shape and complex appearance. Int. J. Comput. Vision, 63(3):175-189.

Kim, J., Kolmogorov, V., and Zabih, R. (2003). "visual correspondence using energy minimization and mutual information".

Klinker, G., Shafer, S., and Kanade, T. (1988). The measurement of highlights in color images. Int. Journal of Computer Vision, 2(1):7-32. 
Koudelka, M., Magda, S., Belhumeur, P., and Kriegman, D. (2001). Image-based modeling and rendering of surfaces with arbitrary BRDFs. In Proc. IEEE Conf. Computer Vision and Pattern Recognition, pages 568-575.

Lee, H. C., Breneman, E. J., and Schulte, C. P. (1990). Modeling light relfection for computer color vision. IEEE Trans. Pattern Analysis and Machine Intelligence, 12(4):402-409.

Lee, H.-S. (1986). Method for computing the scene-illuminant chromaticity from specular highlights. J. Optical Society of America A, 3(10):1694-1699.

Lehmann, T. M. and Palm, C. (2001). Color line search for illuminant estimation in real-world scenes. J. Optical Society of America A, 18(11):2679-2691.

Li, Y., Lin, S., Lu, H., Kang, S. B., and Shum, H.-Y. (2002). Multibaseline stereo in the presence of specular reflections. In ICPR '02: Proceedings of the 16 th International Conference on Pattern Recognition (ICPR'02) Volume 3, page 30573, Washington, DC, USA. IEEE Computer Society.

Lim, J., Ho, J., Yang, M.-H., and Kriegman, D. (2005). Passive photometric stereo from motion. In Proc. IEEE Int. Conf. Computer Vision.

Lin, S., Li, Y., Kang, S. B., Tong, X., and Shum, H.-Y. (2002). Diffuse-specular separation and depth recovery from image sequences. In ECCV '02: Proceedings of the 7th European Conference on Computer Vision-Part III, pages 210224, London, UK. Springer-Verlag.

Lu, J. and Little, J. (1999). Reflectance and shape from images using a collinear light source. Int. Journal of Computer Vision, 32(3):1-28.

Magda, S., Kriegman, D., Zickler, T., and Belhumeur, P. (2001). Beyond Lambert: Reconstructing surfaces with arbitrary BRDFs. In Proc. IEEE Int. Conf. Computer Vision, pages II: 391-398.

Mallick, S. P., Zickler, T. E., Belhumeur, P. N., and Kriegman, D. J. (2006). Specularity Removal In Images and Videos: A PDE Approach. In Proc. European Conf. Computer Vision.

Narasimhan, S. G., Ramesh, V., and Nayar, S. K. (2003). A class of photometric invariants: Separating material from shape and illumination. In Proc. IEEE Int. Conf. Computer Vision, volume 2, pages 1387-1394.

Nayar, S., Fang, X., and Boult, T. (1997). Separation of reflection components using color and polarization. Int. Journal of Computer Vision, 21(3):163-186. 
Nayar, S., Ikeuchi, K., and Kanade, T. (1990). Determining shape and reflectance of hybrid surfaces by photometric sampling. IEEE J. of Robotics and Automation, 6(4):418-431.

Nayar, S. K. and Bolle, M. (1996). Reflectance based object recognition. Int. Journal of Computer Vision, 17(3):219-240.

Park, J. B. (2003). Efficient color representation for image segmentation under nonwhite illumination. In SPIE, Volume 5267, pages 163-174.

Ragheb, H. and Hancock, E. (2001). Separating lambertian and specular reflectance components using iterated conditional modes. In Proc. British Machine Vision Conference, pages 541-522.

Rosenberg, C., Hebert, M., and Thrun, S. (2001). Color constancy using KLdivergence. In Proc. IEEE Int. Conf. Computer Vision, pages 239-247.

Sapiro, G. (1999). Color and illumination voting. IEEE Trans. Pattern Analysis and Machine Intelligence, 21:1210-1215.

Sato, Y. and Ikeutchi, K. (1994). Temporal-color space analysis of reflection. J. Optical Society of America A, 11(11):2990-3002.

Schlüns, K. and Wittig, O. (1993). Photometric stereo for non-Lambertian surfaces using color information. In Proc. Int. Conf. on Image Analysis and Processing, pages 505-512.

Shafer, S. (1985). Using color to separate reflection components. COLOR research and applications, 10(4):210-218.

Silver, W. (1980). Determining shape and reflectance using multiple images. Master's thesis, MIT.

Tagare, H. and deFigueiredo, R. (1991). A theory of photometric stereo for a class of diffuse non-lambertian surfaces. IEEE Trans. Pattern Analysis and Machine Intelligence, 13(2):133-152.

Tan, P., Lin, S., and Quan, L. (2006). Separation of highlight reflections on textured surfaces. In Proc. IEEE Conf. Computer Vision and Pattern Recognition.

Tan, R. T. and Ikeuchi, K. (2003). Separating reflection components of textured surface using a single image. In Proc. IEEE Int. Conf. Computer Vision, pages 870-877. 
Tan, R. T., Nishino, K., and Ikeutchi, K. (2004). Color constancy through inverseintensity chromaticity space. J. Optical Society of America A, 21(3):321-334.

Tian, Y. and Tsui, H. (1997). Shape recovery from a color image for non-lambertian surfaces. J. Optical Society of America A, 14(2):397-404.

Tominga, S. and Wandell, B. (1989). Standard surface-reflectance model and illuminant estimation. J. Optical Society of America A, 6(4):576-584.

Tominga, S. and Wandell, B. A. (2002). Natural scene-illuminant estimation using sensor correlation. Proc. IEEE, 90:42-56.

Tsumura, N., Ojima, N., Sato, K., Shiraishi, M., Shimizu, H., Nabeshima, H., Akazaki, S., Hori, K., and Miyake, Y. (2003). Image-based skin color and texture analysis/synthesis by extracting hemoglobin and melanin information in the skin. In Proc. ACM SIGGRAPH, pages 770-779.

Tu, P. and Mendonça, P. (2003). Surface reconstruction via Helmholtz reciprocity with a single image pair. In Proc. IEEE Conf. Computer Vision and Pattern Recognition, volume 1, pages 541-547.

van de Weijer, J. and Gevers, T. (2004). Robust optical flow from photometric invariants. In Proc. IEEE Int. Conf. Image Processing, pages 1835-1838.

Wann Jensen, H., Marschner, S., Levoy, M., and Hanrahan, P. (2001). A practical model for subsurface light transport. In Proc. ACM SIGGRAPH, pages 511-518.

Wolff, L. and Angelopoulou, E. (1994). Three-dimensional stereo by photometric ratios. J. Optical Society of America A, 11:3069-3078.

Wolff, L. B. and Boult, T. E. (1991). Constraining object features using a polarization reflectance model. IEEE Trans. Pattern Analysis and Machine Intelligence, 13(7):635-657.

Woodham, R. (1978). Photometric stereo: A reflectance map technique for determining surface orientation from image intesity. In Proc. SPIE, volume 155, pages 136-143.

Yang, R., Pollefeys, M., and Welch, G. (2003). Dealing with textureless regions and specular highlights-a progressive space carving scheme using a novel photoconsistency measure. In ICCV '03: Proceedings of the Ninth IEEE International Conference on Computer Vision, page 576, Washington, DC, USA. IEEE Computer Society. 
Yoon, K. and Kweon, I. (2006a). Correspondence search in the presence of specular highlights using specular-free two-band images. In Proc. Asian Conf. on Computer Vision, pages II:761-770.

Yoon, K.-J. and Kweon, I. S. (2006b). Adaptive support-weight approach for correspondence search. IEEE Trans. Pattern Analysis and Machine Intelligence, 28(4):650-656.

Zhang, L., Curless, B., Hertzmann, A., and Seitz, S. M. (2003). Shape and motion under varying illumination: Unifying structure from motion, photometric stereo, and multi-view stereo. In Proc. IEEE Int. Conf. Computer Vision, pages 618625 .

Zheng, Q. and Chellappa, R. (1991). Estimation of illuminant direction, albedo, and shape from shading. IEEE Trans. Pattern Analysis and Machine Intelligence, 13(7):680-702.

Zickler, T., Belhumeur, P., and Kriegman, D. (2002). Helmholtz stereopsis: Exploiting reciprocity for surface reconstruction. In Proc. European Conf. Computer Vision, pages III: 869-884.

Zickler, T. E., Ho, J., Kriegman, D. J., Ponce, J., and Belhumeur, P. N. (2003). Binocular helmholtz stereopsis. In Proc. IEEE Int. Conf. Computer Vision, page 1411, Washington, DC, USA. IEEE Computer Society. 